\title{
CONVERGENCE ANALYSIS OF A RESIDUAL LOCAL PROJECTION FINITE ELEMENT METHOD FOR THE NAVIER-STOKES EQUATIONS*
}

\author{
RODOLFO ARAYA ${ }^{\dagger}$, GABRIEL R. BARRENECHEA ${ }^{\ddagger}$, ABNER H. POZA $^{\S}$, AND \\ FRÉDÉRIC VALENTIN
}

\begin{abstract}
This work presents and analyzes a new residual local projection stabilized finite element method (RELP) for the nonlinear incompressible Navier-Stokes equations. Stokes problems defined elementwise drive the construction of the residual-based terms which make the present method stable for the finite element pairs $\mathbb{P}_{1} / \mathbb{P}_{l}, l=0,1$. Numerical upwinding is incorporated through an extra control on the advective derivative and on the residual of the divergence equation. Well-posedness of the discrete problem as well as optimal error estimates in natural norms are proved under standard assumptions. Next, a divergence-free velocity field is provided by a simple postprocessing of the computed velocity and pressure using the lowest order Raviart-Thomas basis functions. This updated velocity is proved to converge optimally to the exact solution. Numerics assess the theoretical results and validate the RELP method.
\end{abstract}

Key words. Navier-Stokes equations, RELP method, stabilized low order method, numerical analysis

AMS subject classifications. $65 \mathrm{~N} 12,65 \mathrm{~N} 15,65 \mathrm{~N} 30$

DOI. $10.1137 / 110829283$

1. Introduction. The numerical solution of the incompressible Navier-Stokes equations by standard finite element methods demands the selection of inf-sup stable pairs of interpolation spaces for the velocity and the pressure [17]. This condition prevents the most desirable choices of spaces to be adopted, such as the simplest and lowest equal order elements [12]. Also, numerical methods should include upwinding strategies to avoid spurious oscillations when the exact solution develops boundary layers [20], behavior that appears for high Reynolds numbers flows.

In the quest to bypass these issues, a branch of new stabilized finite element methods, called local projection stabilized (LPS) methods, have been recently introduced for mixed problems (see $[4,11]$ ). Like classical stabilized methods, the main idea lies in the addition of extra terms to the Galerkin method but with the difference

*Received by the editors April 1, 2011; accepted for publication (in revised form) December 12, 2011; published electronically April 10, 2012. Part of this work was carried out during the first and second authors' stay at the Departamento de Matemática Aplicada e Computacional, Laboratório Nacional de Computação Científica, Brazil, which was partially funded by a PCI/MCT grant.

http://www.siam.org/journals/sinum/50-2/82928.html

${ }^{\dagger} \mathrm{CI}^{2} \mathrm{MA}$ and Departamento de Ingeniería Matemática, Universidad de Concepción, Casilla 160C, Concepción, Chile (raraya@ing-mat.udec.cl). This author's work was partially supported by CONICYT/Chile through FONDECYT project 1070698 and Basal project CMM-CI2MA PFB-03.

${ }^{\ddagger}$ Department of Mathematics and Statistics, University of Strathclyde, 26 Richmond Street, Glasgow G1 1XH, Scotland (gabriel.barrenechea@strath.ac.uk). This author's work was partially funded by the London Mathematical Society through LMS grant 41003.

$\S$ Departamento de Ingeniería Matemática, Universidad de Concepción, Casilla 160-C, Concepción, Chile (abner@ing-mat.udec.cl). This author's work was partially funded by CONICYT/Chile through a scholarship program and by FONDECYT project 1070698.

๑ Departamento de Matemática Aplicada e Computacional, Laboratório Nacional de Computação Científica, Av. Getúlio Vargas, 333, 25651-070 Petrópolis - RJ, Brazil (valentin@lncc.br). This author's work was supported by CONICYT/Chile through FONDECYT project 7100007 and CNPq/Brazil through grants 302730/2009-5 and 475543/2009-2.

669 
that they are no longer dependent on residuals but rather are constructed using the fluctuation between the variables (or their derivatives) and their projection onto a given finite dimensional space. (See [15] for an interesting overview.) LPS methods have been extended to the Oseen model in [6,7], although their capacity to handle singularly perturbed models still deserves more investigation [18].

Also recently, a relationship between enriching polynomial spaces with the solution of local problems and LPS methods has been established (see [14, 1]). The resulting methods, called residual local projection (RELP) stabilized methods, reintroduced residuals as the main ingredient in their construction but now they are included through fluctuation operators. Thereby, some of the standard LPS extra terms can be seen as a consequence of an enriching space procedure. Next, a new RELP method [2] has been proposed for the Oseen equation and validated for advection dominated flows. Also, a simplified version of the method, specially suited for lower order methods, was presented in [3]. Well-posedness and optimal error estimates were obtained for both methods, which were extensively validated through singularly perturbed benchmarks.

In this work, we extend the RELP method to the nonlinear incompressible NavierStokes equations. We seek the method bearing in mind a certain set of desired characteristics, including the following:

- Be stable and achieve optimal convergent in natural norms for $\mathbb{P}_{1} / \mathbb{P}_{l}, l=0,1$;

- Bring balanced numerical diffusion;

- Be easily postprocessed such as the discrete solution is divergence-free.

To address these requirements, the method is developed within the framework proposed in [1] in which boundary value problems account for residuals at the element level, which ultimately are responsible for stabilizing the Galerkin method. In particular, we choose to set up a Stokes model element wisely. This accounts for diffusive processes that dominate flows at small scales and might be modeled through the residuals at large scales. Now, since no analytical solution is available for this local problem, a two-level numerical strategy is needed to implement the method which makes the approach more involved. In view of making the present method attractive for practitioners, we project the residual onto the space of piecewise constant functions before solving the local problem. This simplification makes the local problem analytically solvable and provides an alternative RELP method that does not undermine the convergence of the method. In the process, a way to construct an exactly divergence-free velocity field is also set up.

The stability and convergence analysis of the RELP method is based on the fixed point theory presented in $[8,17]$ and used in $[24]$ to analyze the SDFEM method originally proposed in [13] in the case where diffusion dominates and in [10] for a pressure-stabilized finite element method. Here, we extend that idea and prove that the original RELP method is well-posed and achieve optimal convergence in natural norms. Due to the particular structure of the method, the proof requests the construction of a new stabilized finite element method for the Stokes equation, related to the one given in [1], which is also analyzed. Finally, we establish that the postprocessed divergence-free velocity field is also optimally convergent.

The paper is outlined as follows. We end this section with some notation and definitions to be used throughout this manuscript. The next section is devoted to the presentation of the RELP methods. Section 3 includes a well-posedness result and error estimates and the postprocessing to get a divergence-free velocity field. Numerical validations are in section 4 , and some conclusions are drawn in section 5 . Finally, in Appendix A we investigate numerical aspects of a new stabilized method 
for the Stokes model, and in Appendix B we give a detailed prove of the uniqueness of solution for the RELP method.

1.1. Notation and preliminaries. Let $\Omega \subseteq \mathbb{R}^{2}$ be a polygonal open domain. The steady incompressible Navier-Stokes equations consist of finding the velocity and pressure $(\boldsymbol{u}, \tilde{p})$ as the solution of

$$
\begin{gathered}
-\nu \Delta \boldsymbol{u}+(\nabla \boldsymbol{u}) \boldsymbol{u}+\nabla \tilde{p}=\tilde{\boldsymbol{f}}, \quad \nabla \cdot \boldsymbol{u}=0 \quad \text { in } \Omega, \\
\boldsymbol{u}=\mathbf{0} \quad \text { on } \partial \Omega,
\end{gathered}
$$

where $\nu \in \mathbb{R}^{+}$is the fluid viscosity and $\tilde{\boldsymbol{f}} \in L^{2}(\Omega)^{2}$. Adopting standard notation for Sobolev spaces, the weak form associated to (1.1) reads, Find $(\boldsymbol{u}, \tilde{p}) \in$ $\boldsymbol{V} \times Q:=H_{0}^{1}(\Omega)^{2} \times L_{0}^{2}(\Omega)$ such that

$$
\nu(\nabla \boldsymbol{u}, \nabla \boldsymbol{v})+((\nabla \boldsymbol{u}) \boldsymbol{u}, \boldsymbol{v})-(\tilde{p}, \nabla \cdot \boldsymbol{v})+(q, \nabla \cdot \boldsymbol{u})=(\tilde{\boldsymbol{f}}, \boldsymbol{v}) \quad \forall(\boldsymbol{v}, q) \in \boldsymbol{V} \times Q,
$$

where $(\cdot, \cdot)$ stands for the $L^{2}(\Omega)$-inner product, where we use the same notation for vector, or tensor, valued functions.

We remark that the continuous problem (1.2) has at least one solution, which is unique in the small data case. (See Theorem 2.4 in [17] for the details.)

Let $D$ be an open subset of $\Omega$. We denote by $\|\cdot\|_{m, D}$ the norm in $H^{m}(D)$ and by $\|\cdot\|_{m, q, D}$ the norm in $W^{m, q}(D)$ with $m \geq 0$ and $1 \leq q \leq \infty$. We denote, as usual, $H^{-1}(\Omega)$ the dual space of $H_{0}^{1}(\Omega)$ equipped with the dual norm $\|\cdot\|_{-1, \Omega}$ and the duality product $\langle\cdot, \cdot\rangle$, and $H^{0}(\Omega)=L^{2}(\Omega)$ and $W^{0, q}(\Omega)=L^{q}(\Omega)$. Also, we define the norm $\|\cdot\|$, in $\boldsymbol{V} \times Q$, by

$$
\|(\boldsymbol{v}, q)\|:=\left\{|\boldsymbol{v}|_{1, \Omega}^{2}+\|q\|_{0, \Omega}^{2}\right\}^{1 / 2}
$$

and the norm $\|\cdot\|_{(\boldsymbol{V} \times Q)^{\prime}}$ in $(\boldsymbol{V} \times Q)^{\prime}$, the dual space of $\boldsymbol{V} \times Q$, by

$$
\|(\boldsymbol{v}, q)\|_{(\boldsymbol{V} \times Q)^{\prime}}:=\sup _{\|(\boldsymbol{w}, r)\| \leq 1}\{\langle\boldsymbol{v}, \boldsymbol{w}\rangle+(q, r)\} .
$$

Let $\left\{\mathcal{T}_{h}\right\}_{h>0}$ be a family of regular triangulations of $\Omega$, built up using triangles $K$ with boundary $\partial K$ and characteristic length $h_{K}:=\operatorname{diam}(K)$ and $h:=\max \left\{h_{K}: K \in\right.$ $\mathcal{T}_{h}$ \}. The set of internal edges $F$ of the triangulation is denoted $\mathcal{E}_{h}$ with $h_{F}=|F|$. We denote by $\boldsymbol{n}$ the normal outward vector on $\partial K$; also, $\llbracket v \rrbracket$ stands for the jump of $v$ across $F$. In addition, for $K \in \mathcal{T}_{h}$ and $F \in \mathcal{E}_{h}$, we define the neighborhoods $\omega_{K}=\left\{K^{\prime} \in \mathcal{T}_{h}: K^{\prime} \cap K \neq \emptyset\right\}$ and $\omega_{F}=\left\{K \in \mathcal{T}_{h}: F \cap K \neq \emptyset\right\}$. Finally, we denote by $\Pi_{S}$, where $S \subset \mathbb{R}^{2}$, the orthogonal projection onto the constant space, i.e., $\Pi_{S}(q):=\frac{(q, 1)_{S}}{|S|}$, and by $H^{m}\left(\mathcal{T}_{h}\right), m \geq 1$, we denote the space of functions whose restriction to $K \in \mathcal{T}_{h}$ belongs to $H^{m}(K)$.

Associated to the triangulation $\mathcal{T}_{h}$, the discrete space for the velocity $\boldsymbol{V}_{h}$ is the usual space of vector valued continuous piecewise linear functions with zero trace on $\partial \Omega$. To approximate the pressure we use $Q_{h}$, the space of piecewise polynomial functions of degree $l,(l=0,1)$ with zero mean value on $\Omega$. If $l=1$, the space of pressures may contain continuous or discontinuous functions. Analogous to (1.3) we introduce the following norm in the dual of the discrete space:

$$
\|(\boldsymbol{v}, q)\|_{\left(\boldsymbol{V}_{h} \times Q_{h}\right)^{\prime}}:=\sup _{\left\|\left(\boldsymbol{w}_{h}, r_{h}\right)\right\| \leq 1}\left\{\left\langle\boldsymbol{v}, \boldsymbol{w}_{h}\right\rangle+\left(q, r_{h}\right)\right\} .
$$


In what follows, we will employ the differential of a mapping $F: \boldsymbol{V} \times Q \rightarrow \boldsymbol{V} \times Q$ with respect to $(\boldsymbol{u}, q)$ at $(\boldsymbol{v}, q) \in \boldsymbol{V} \times Q$ denoted by $D_{\boldsymbol{u}, p} F(\boldsymbol{v}, q) \in \mathcal{L}(\boldsymbol{V} \times Q)$, where $\mathcal{L}(\boldsymbol{V} \times Q)$ represents the space of linear mappings acting on elements of $\boldsymbol{V} \times Q$ with values in $\boldsymbol{V} \times Q$ and equipped with the usual norm $\|\cdot\|_{\mathcal{L}(\boldsymbol{V} \times Q)}$.

Finally, in the forthcoming analysis we will use the following classical result.

Lemma 1.1. For all $\boldsymbol{v}, \boldsymbol{w} \in \boldsymbol{V}$ and $q \in Q$, we have

$$
\begin{aligned}
& \sup _{\boldsymbol{v} \in \boldsymbol{V}} \frac{(q, \nabla \cdot \boldsymbol{v})}{|\boldsymbol{v}|_{1, \Omega}} \geq \beta\|q\|_{0, \Omega}, \\
& \quad((\nabla \boldsymbol{u}) \boldsymbol{w}, \boldsymbol{v}) \leq \alpha|\boldsymbol{u}|_{1, \Omega}|\boldsymbol{w}|_{1, \Omega}|\boldsymbol{v}|_{1, \Omega},
\end{aligned}
$$

where $\alpha$ and $\beta$ are positive constants depending only on $\Omega$. Moreover, for all $\boldsymbol{u}, \boldsymbol{v}, \boldsymbol{w} \in$ $\boldsymbol{V}$, it holds that

$$
\begin{aligned}
& ((\nabla \boldsymbol{u}) \boldsymbol{w}, \boldsymbol{v})=-((\nabla \boldsymbol{v}) \boldsymbol{w}, \boldsymbol{u})-(\nabla \cdot \boldsymbol{w}, \boldsymbol{u} \cdot \boldsymbol{v}) \\
& ((\nabla \boldsymbol{v}) \boldsymbol{w}, \boldsymbol{v})=-\frac{1}{2}(\nabla \cdot \boldsymbol{w}, \boldsymbol{v} \cdot \boldsymbol{v})
\end{aligned}
$$

Proof. See [17] and [22].

2. The residual local projection method. The finite element method that we analyze in this work reads, Find $\left(\boldsymbol{u}_{h}, \tilde{p}_{h}\right) \in \boldsymbol{V}_{h} \times Q_{h}$ such that

$$
\begin{aligned}
\nu\left(\nabla \boldsymbol{u}_{h}, \nabla \boldsymbol{v}_{h}\right)+ & \left(\left(\nabla \boldsymbol{u}_{h}\right) \boldsymbol{u}_{h}, \boldsymbol{v}_{h}\right)-\left(\tilde{p}_{h}, \nabla \cdot \boldsymbol{v}_{h}\right)+\left(\tilde{q}_{h}, \nabla \cdot \boldsymbol{u}_{h}\right) \\
+ & +\sum_{K \in \mathcal{T}_{h}}\left[\frac{\alpha_{K}}{\nu}\left(p_{e}^{K}\left(-\tilde{\boldsymbol{f}}-\Delta \boldsymbol{u}_{h}+\left(\nabla \boldsymbol{u}_{h}\right) \boldsymbol{u}_{h}+\nabla \tilde{p}_{h}\right), p_{e}^{K}\left(\left(\nabla \boldsymbol{v}_{h}\right) \boldsymbol{u}_{h}+\nabla \tilde{q}_{h}\right)\right)_{K}\right. \\
& \left.+\frac{\gamma_{K}}{\nu}\left(\chi_{h}\left(\boldsymbol{x} \nabla \cdot \boldsymbol{u}_{h}\right), \chi_{h}\left(\boldsymbol{x} \nabla \cdot \boldsymbol{v}_{h}\right)\right)_{K}\right] \\
+ & \sum_{F \in \mathcal{E}_{h}} \tau_{F}\left(\llbracket \nu \partial_{\boldsymbol{n}} \boldsymbol{u}_{h}+\tilde{p}_{h} \boldsymbol{n} \rrbracket, \llbracket \nu \partial_{\boldsymbol{n}} \boldsymbol{v}_{h}+\tilde{q}_{h} \boldsymbol{n} \rrbracket\right)_{F}=\left(\tilde{\boldsymbol{f}}, \boldsymbol{v}_{h}\right)
\end{aligned}
$$

for all $\left(\boldsymbol{v}_{h}, \tilde{q}_{h}\right) \in \boldsymbol{V}_{h} \times Q_{h}$, where $\chi_{h}:=I-\Pi_{K}$ is the fluctuation operator.

Here, for a function $\boldsymbol{v} \in L^{2}(K)^{2},\left(\boldsymbol{u}_{e}^{K}(\boldsymbol{v}), p_{e}^{K}(\boldsymbol{v})\right)$ stands for the solution of the local Stokes problem

$$
\begin{aligned}
& -\nu \Delta \boldsymbol{u}_{e}^{K}(\boldsymbol{v})+\nabla p_{e}^{K}(\boldsymbol{v})=\boldsymbol{v}, \quad \nabla \cdot \boldsymbol{u}_{e}^{K}(\boldsymbol{v})=0 \quad \text { in } K, \\
& \boldsymbol{u}_{e}^{K}(\boldsymbol{v})=\mathbf{0} \quad \text { on } \partial K \text {. }
\end{aligned}
$$

Also, the stabilization parameters are given by

$$
\alpha_{K}:=\frac{1}{\max \left\{1, P e_{K}\right\}} \quad \text { and } \quad \gamma_{K}:=\frac{1}{\max \left\{1, \frac{P e_{K}}{24}\right\}},
$$

where

$$
P e_{K}:=\frac{\left|\boldsymbol{u}_{h}\right|_{K} h_{K}}{18 \nu} \quad \text { with } \quad\left|\boldsymbol{u}_{h}\right|_{K}:=\frac{\left\|\boldsymbol{u}_{h}\right\|_{0, K}}{|K|^{1 / 2}}
$$

and

$$
\tau_{F}:= \begin{cases}\frac{h_{F}}{12 \nu} & \text { if }\left|\boldsymbol{u}_{h}\right|_{F}=0 \\ \frac{1}{2\left|\boldsymbol{u}_{h}\right|_{F}}-\frac{1}{\left|\boldsymbol{u}_{h}\right|_{F}\left(1-\exp \left(P e_{F}\right)\right)}\left(1+\frac{1}{P e_{F}}\left(1-\exp \left(P e_{F}\right)\right)\right) & \text { otherwise. }\end{cases}
$$


Here

$$
P e_{F}:=\frac{\left|\boldsymbol{u}_{h}\right|_{F} h_{F}}{\nu} \quad \text { with } \quad\left|\boldsymbol{u}_{h}\right|_{F}:=\frac{\left\|\boldsymbol{u}_{h}\right\|_{0, F}}{h_{F}^{1 / 2}}
$$

In general (2.1) requests a two-level discretization since problem (2.2) cannot be exactly solved. On the other hand, a closer inspection of problem (2.2) reveals that some terms in (2.1) can indeed be exactly computed, thus simplifying the implementation. In particular, we realize that for all $q \in H^{1}(K)$ it holds that

$$
\boldsymbol{u}_{e}^{K}(\nabla q)=\mathbf{0} \quad \text { and } \quad p_{e}^{K}(\nabla q)=\chi_{h}(q) .
$$

As for the remaining terms, we replace $p_{e}^{K}\left(-\tilde{\boldsymbol{f}}+\left(\nabla \boldsymbol{u}_{h}\right) \boldsymbol{u}_{h}+\nabla \tilde{p}_{h}\right)$ by $p_{e}^{K}\left(\Pi_{K}(-\tilde{\boldsymbol{f}}+\right.$ $\left.\left.\left(\nabla \boldsymbol{u}_{h}\right) \boldsymbol{u}_{h}+\nabla \tilde{p}_{h}\right)\right)$ and $p_{e}^{K}\left(\left(\nabla \boldsymbol{v}_{h}\right) \boldsymbol{u}_{h}+\nabla q_{h}\right)$ by $p_{e}^{K}\left(\Pi_{K}\left(\left(\nabla \boldsymbol{v}_{h}\right) \boldsymbol{u}_{h}+\nabla q_{h}\right)\right)$ in (2.1) to get the following simplified method: Find $\left(\boldsymbol{u}_{h}, \tilde{p}_{h}\right) \in \boldsymbol{V}_{h} \times Q_{h}$ such that

$$
\begin{aligned}
& \nu\left(\nabla \boldsymbol{u}_{h}, \nabla \boldsymbol{v}_{h}\right)+\left(\left(\nabla \boldsymbol{u}_{h}\right) \boldsymbol{u}_{h}, \boldsymbol{v}_{h}\right)-\left(\tilde{p}_{h}, \nabla \cdot \boldsymbol{v}_{h}\right)+\left(q_{h}, \nabla \cdot \boldsymbol{u}_{h}\right) \\
& \quad+\sum_{K \in \mathcal{T}_{h}} \frac{\alpha_{K}}{\nu}\left(p_{e}^{K}\left(\Pi_{K}\left(-\tilde{\boldsymbol{f}}+\left(\nabla \boldsymbol{u}_{h}\right) \boldsymbol{u}_{h}+\nabla \tilde{p}_{h}\right)\right), p_{e}^{K}\left(\Pi_{K}\left(\left(\nabla \boldsymbol{v}_{h}\right) \boldsymbol{u}_{h}+\nabla q_{h}\right)\right)\right)_{K} \\
& \quad+\sum_{K \in \mathcal{T}_{h}} \frac{\gamma_{K}}{\nu}\left(\chi_{h}\left(\boldsymbol{x} \nabla \cdot \boldsymbol{u}_{h}\right), \chi_{h}\left(\boldsymbol{x} \nabla \cdot \boldsymbol{v}_{h}\right)\right)_{K} \\
& \quad+\sum_{F \in \mathcal{E}_{h}} \tau_{F}\left(\llbracket \nu \partial_{\boldsymbol{n}} \boldsymbol{u}_{h}+\tilde{p}_{h} \boldsymbol{n} \rrbracket, \llbracket \nu \partial_{\boldsymbol{n}} \boldsymbol{v}_{h}+q_{h} \boldsymbol{n} \rrbracket\right)_{F}=\left(\tilde{\boldsymbol{f}}, \boldsymbol{v}_{h}\right) .
\end{aligned}
$$

Finally, noting that every constant is a gradient, we apply (2.3) to derive the final form of the simplified RELP method: Find $\left(\boldsymbol{u}_{h}, \tilde{p}_{h}\right) \in \boldsymbol{V}_{h} \times Q_{h}$ such that

$$
\begin{aligned}
& \nu\left(\nabla \boldsymbol{u}_{h}, \nabla \boldsymbol{v}_{h}\right)+\left(\left(\nabla \boldsymbol{u}_{h}\right) \boldsymbol{u}_{h}, \boldsymbol{v}_{h}\right)-\left(\tilde{p}_{h}, \nabla \cdot \boldsymbol{v}_{h}\right)+\left(q_{h}, \nabla \cdot \boldsymbol{u}_{h}\right) \\
& \quad+\sum_{K \in \mathcal{T}_{h}} \frac{\alpha_{K}}{\nu}\left(\chi_{h}\left(\boldsymbol{x} \cdot\left(\nabla \boldsymbol{u}_{h}\right) \Pi_{K} \boldsymbol{u}_{h}+\tilde{p}_{h}\right), \chi_{h}\left(\boldsymbol{x} \cdot\left(\nabla \boldsymbol{v}_{h}\right) \Pi_{K} \boldsymbol{u}_{h}+q_{h}\right)\right)_{K} \\
& \quad+\sum_{K \in \mathcal{T}_{h}} \frac{\gamma_{K}}{\nu}\left(\chi_{h}\left(\boldsymbol{x} \nabla \cdot \boldsymbol{u}_{h}\right), \chi_{h}\left(\boldsymbol{x} \nabla \cdot \boldsymbol{v}_{h}\right)\right)_{K} \\
& \quad+\sum_{F \in \mathcal{E}_{h}} \tau_{F}\left(\llbracket \nu \partial_{\boldsymbol{n}} \boldsymbol{u}_{h}+\tilde{p}_{h} \boldsymbol{n} \rrbracket, \llbracket \nu \partial_{\boldsymbol{n}} \boldsymbol{v}_{h}+q_{h} \boldsymbol{n} \rrbracket\right)_{F} \\
& =\left(\tilde{\boldsymbol{f}}, \boldsymbol{v}_{h}\right)+\sum_{K \in \mathcal{T}_{h}} \frac{\alpha_{K}}{\nu}\left(\chi_{h}\left(\boldsymbol{x} \cdot \Pi_{K} \tilde{\boldsymbol{f}}\right), \chi_{h}\left(\boldsymbol{x} \cdot\left(\nabla \boldsymbol{v}_{h}\right) \Pi_{K} \boldsymbol{u}_{h}+q_{h}\right)\right)_{K} .
\end{aligned}
$$

Remark 1.

- Both methods (2.1) and (2.4) can be seen as the Galerkin method enhanced with stabilizing terms. These terms can be split as the sum of two local pressures coming from the solution of local Stokes problems (with the residual on the right-hand side), plus a fluctuation term mimicking a grad-div stabilizing term, plus a jump term aiming at stabilizing the discontinuities of the pressure. In case the interpolation space for the pressure is continuous, this jump term becomes superfluous and can be omitted.

- The method (2.4) is the one we will implement since it is not a two-level method. The next section deals with the error analysis for the original RELP method (2.1), as the analysis can be extended, with minor differences, to the simplified RELP method (2.4). The reason to analyze method (2.1) is its 
generality, which opens the door to different solution strategies for the local problem (2.2), and it can be seen as the first steps toward the analysis of general two-level finite element methods for the Navier-Stokes equations. The two-level approach is a particularly attractive option when solutions involve multiple scales as occurring in porous media or turbulent flows, for instance, as the alternative of using a very fine mesh becomes prohibitive.

- The design of the stabilization parameters $\alpha_{K}, \gamma_{K}$, and $\tau_{F}$ has been motivated by the enrichment of the finite element space presented in [2], where analogous choices were shown to be the ones providing the best results for the Oseen equation. In fact, the RELP method appeared to be very competitive when compared to more classical stabilized methods, which motivates its use for the nonlinear Navier-Stokes equations.

- The methods (2.1) and (2.4) remain unchanged in the three-dimensional setting and in case higher order finite element spaces are used.

3. Error analysis. We consider a scaled form of (1.1) by setting $\tilde{p}=\nu p, \tilde{\boldsymbol{f}}=$ $\nu \boldsymbol{f}$, and $\lambda=\nu^{-1}$ and rewrite (1.1) as

$$
\begin{gathered}
-\Delta \boldsymbol{u}_{\lambda}+\lambda\left(\nabla \boldsymbol{u}_{\lambda}\right) \boldsymbol{u}_{\lambda}+\nabla p_{\lambda}=\boldsymbol{f}, \quad \nabla \cdot \boldsymbol{u}_{\lambda}=0 \quad \text { in } \Omega, \\
\boldsymbol{u}_{\lambda}=\mathbf{0} \quad \text { on } \partial \Omega .
\end{gathered}
$$

The standard weak formulation of problem (3.1) is given by the following: Find $\left(\boldsymbol{u}_{\lambda}, p_{\lambda}\right) \in \boldsymbol{V} \times Q$ such that

$$
\left(\nabla \boldsymbol{u}_{\lambda}, \nabla \boldsymbol{v}\right)+\lambda\left(\left(\nabla \boldsymbol{u}_{\lambda}\right) \boldsymbol{u}_{\lambda}, \boldsymbol{v}\right)-\left(p_{\lambda}, \nabla \cdot \boldsymbol{v}\right)+\left(q, \nabla \cdot \boldsymbol{u}_{\lambda}\right)=(\boldsymbol{f}, \boldsymbol{v}) \quad \forall(\boldsymbol{v}, q) \in \boldsymbol{V} \times Q .
$$

We assume in this work that problem (3.2) admits at least one solution, which is unique provided $\lambda$ is sufficiently small. Also, (3.2) can be written in the operator form as

$$
F\left(\lambda, \boldsymbol{u}_{\lambda}, p_{\lambda}\right):=\left(\boldsymbol{u}_{\lambda}, p_{\lambda}\right)+T G\left(\lambda, \boldsymbol{u}_{\lambda}, p_{\lambda}\right)=\mathbf{0},
$$

where $G\left(\lambda, \boldsymbol{u}_{\lambda}, p_{\lambda}\right) \in \boldsymbol{V}^{\prime} \times Q$ is given by

$$
\left\langle G\left(\lambda, \boldsymbol{u}_{\lambda}, p_{\lambda}\right),(\boldsymbol{v}, q)\right\rangle:=\lambda\left(\left(\nabla \boldsymbol{u}_{\lambda}\right) \boldsymbol{u}_{\lambda}, \boldsymbol{v}\right)-(\boldsymbol{f}, \boldsymbol{v}) \quad \forall(\boldsymbol{v}, q) \in \boldsymbol{V} \times Q,
$$

and $T: \boldsymbol{V}^{\prime} \times Q \longrightarrow \boldsymbol{V} \times Q$ denotes the Stokes operator, which associates for each $(\boldsymbol{w}, r) \in \boldsymbol{V}^{\prime} \times Q$ the unique solution $(\boldsymbol{u}, p) \in \boldsymbol{V} \times Q$ of

$$
(\nabla \boldsymbol{u}, \nabla \boldsymbol{v})-(p, \nabla \cdot \boldsymbol{v})+(q, \nabla \cdot \boldsymbol{u})=\langle\boldsymbol{w}, \boldsymbol{v}\rangle+(r, q)
$$

for all $(\boldsymbol{v}, q) \in \boldsymbol{V} \times Q$.

The stabilized method for problem (3.1) reads as follows: Find $\left(\boldsymbol{u}_{h, \lambda}, p_{h, \lambda}\right) \in$ $\boldsymbol{V}_{h} \times Q_{h}$ such that for all $\left(\boldsymbol{v}_{h}, q_{h}\right) \in \boldsymbol{V}_{h} \times Q_{h}$,

$$
\begin{aligned}
& \left(\nabla \boldsymbol{u}_{h, \lambda}, \nabla \boldsymbol{v}_{h}\right)+\lambda\left(\left(\nabla \boldsymbol{u}_{h, \lambda}\right) \boldsymbol{u}_{h, \lambda}, \boldsymbol{v}_{h}\right)-\left(p_{h, \lambda}, \nabla \cdot \boldsymbol{v}_{h}\right)+\left(q_{h}, \nabla \cdot \boldsymbol{u}_{h, \lambda}\right) \\
& \quad+\sum_{K \in \mathcal{T}_{h}} \alpha_{K}\left(p_{e}^{K}\left(-\Delta \boldsymbol{u}_{h, \lambda}+\lambda\left(\nabla \boldsymbol{u}_{h, \lambda}\right) \boldsymbol{u}_{h, \lambda}+\nabla p_{h, \lambda}\right), p_{e}^{K}\left(\lambda\left(\nabla \boldsymbol{v}_{h}\right) \boldsymbol{u}_{h, \lambda}+\nabla q_{h}\right)\right)_{K} \\
& +\sum_{K \in \mathcal{T}_{h}} \gamma_{K}\left(\lambda \chi_{h}\left(\boldsymbol{x} \nabla \cdot \boldsymbol{u}_{h, \lambda}\right), \lambda \chi_{h}\left(\boldsymbol{x} \nabla \cdot \boldsymbol{v}_{h}\right)\right)_{K} \\
& +\sum_{F \in \mathcal{E}_{h}} \tilde{\tau}_{F}\left(\llbracket \partial_{\boldsymbol{n}} \boldsymbol{u}_{h, \lambda}+p_{h, \lambda} \boldsymbol{n} \rrbracket, \llbracket \partial_{\boldsymbol{n}} \boldsymbol{v}_{h}+q_{h} \boldsymbol{n} \rrbracket\right)_{F} \\
& =\left(\boldsymbol{f}, \boldsymbol{v}_{h}\right)+\sum_{K \in \mathcal{T}_{h}} \alpha_{K}\left(p_{e}^{K}(\boldsymbol{f}), p_{e}^{K}\left(\lambda\left(\nabla \boldsymbol{v}_{h}\right) \boldsymbol{u}_{h, \lambda}+\nabla q_{h}\right)\right)_{K},
\end{aligned}
$$


where $\tilde{\tau}_{F}=\frac{\tau_{F}}{\lambda}$ and $p_{e}^{K}$ solves the Stokes local problem (2.2). Multiplying (3.4) by $\nu$, and substituting $\tilde{p}_{h}=\nu p_{h}, \tilde{\boldsymbol{f}}=\nu \boldsymbol{f}$, and $\tilde{q}_{h}=\nu q_{h}$, we recover the RELP method (2.1) for the original, unscaled Navier-Stokes equations.

3.1. Technical preliminaries. We first state the following local trace theorem [23].

LEMma 3.1. There exists a constant $C>0$, independent of $h$, such that for all $v \in H^{1}(K)$

$$
\|v\|_{0, \partial K}^{2} \leq C\left\{h_{K}^{-1}\|v\|_{0, K}^{2}+h_{K}|v|_{1, K}^{2}\right\} .
$$

The following inverse estimates are satisfied for $\boldsymbol{V}_{h}$ and $Q_{h}$. (For a proof see, e.g., [12].)

Lemma 3.2. There exists a constant $C>0$, independent of $h$, such that for all $\boldsymbol{v}_{h} \in \boldsymbol{V}_{h}$ and all $q_{h} \in Q_{h}$ there holds

$$
\begin{aligned}
\left\|\boldsymbol{v}_{h}\right\|_{\infty, K} & \leq C h_{K}^{-1}\left\|\boldsymbol{v}_{h}\right\|_{0, K}, \\
\left\|\llbracket q_{h} \rrbracket\right\|_{0, F} & \leq C h_{F}^{-1 / 2}\left\|q_{h}\right\|_{0, \omega_{F}}, \\
h_{K}\left|\boldsymbol{v}_{h}\right|_{1, K} & \leq C\left\|\boldsymbol{v}_{h}\right\|_{0, K} .
\end{aligned}
$$

The properties of the orthogonal projection $\Pi_{K}$ onto the constant space are summarized in the following result.

LEMma 3.3. There exists a constant $C>0$, independent of $h$, such that

$$
\begin{aligned}
\left\|v-\Pi_{K} v\right\|_{0, K} & \leq C h_{K}|v|_{1, K} \quad \forall v \in H^{1}(K), \\
\left\|\Pi_{K} v\right\|_{0, K} & \leq\|v\|_{0, K} \quad \forall v \in L^{2}(K), \\
\left\|\Pi_{K} v\right\|_{\infty, K} & \leq C h_{K}^{-1}\|v\|_{0, K} \quad \forall v \in L^{2}(K) .
\end{aligned}
$$

Proof. For estimates (3.8) and (3.9), see [12]. Finally, (3.10) follows from (3.5) and (3.9).

We introduce the Lagrange interpolation operator $\mathcal{I}_{h}: \boldsymbol{V} \cap H^{2}(\Omega)^{2} \longrightarrow \boldsymbol{V}_{h}$ and the operator $\mathcal{J}_{h}: Q \longrightarrow Q_{h}$ for the velocity and pressure, respectively, where $\mathcal{J}_{h}$ is a modified Clément operator for continuous pressures $(l=1)$ or the orthogonal projection onto $Q_{h}$ for discontinuous pressures. These interpolation operators satisfy $($ see $[9,12])$

$$
\begin{aligned}
\left|\boldsymbol{v}-\mathcal{I}_{h} \boldsymbol{v}\right|_{m, K} & \leq C h_{K}^{2-m}|\boldsymbol{v}|_{2, K} \quad \forall \boldsymbol{v} \in H^{2}(K)^{2}, \\
\left|\mathcal{I}_{h} \boldsymbol{v}\right|_{1, K} & \leq C\|\boldsymbol{v}\|_{2, K} \quad \forall \boldsymbol{v} \in H^{2}(K)^{2}, \\
\left\|\boldsymbol{v}-\mathcal{I}_{h} \boldsymbol{v}\right\|_{i, F} & \leq C h_{F}^{3 / 2-i}|\boldsymbol{v}|_{2, \omega_{F}} \quad \forall \boldsymbol{v} \in H^{2}\left(\omega_{F}\right)^{2}, \\
\left\|\boldsymbol{v}-\mathcal{I}_{h} \boldsymbol{v}\right\|_{1, r, K} & \leq C\|\boldsymbol{v}\|_{1, r, K} \quad \forall \boldsymbol{v} \in W^{1, r}(K)^{2} \forall r \in(2, \infty], \\
\left\|\boldsymbol{v}-\mathcal{I}_{h} \boldsymbol{v}\right\|_{\infty, K} \leq C\|\boldsymbol{v}\|_{\infty, K} \quad \forall \boldsymbol{v} \in \mathcal{C}^{0}(K)^{2}, & \\
\left|q-\mathcal{J}_{h} q\right|_{i, K} & \leq C h_{K}^{j-i}|q|_{j, \omega_{K}} \quad \forall q \in H^{j}\left(\omega_{K}\right), \\
\left\|q-\mathcal{J}_{h} q\right\|_{0, F} & \leq C h_{F}^{j-1 / 2}|q|_{j, \omega_{F}} \quad \forall q \in H^{j}\left(\omega_{F}\right),
\end{aligned}
$$

where $0 \leq m \leq 2$ and $0 \leq i \leq 1,1 \leq j \leq l+1$, and $C>0$ is independent of $h$.

Before heading to well-posedness results, we give the following technical result, whose proof can be found in [1].

Copyright $@$ by SIAM. Unauthorized reproduction of this article is prohibited. 
Lemma 3.4. Let $\boldsymbol{v} \in L^{2}(K)^{2}$ and let $\left(\boldsymbol{u}_{e}^{K}(\boldsymbol{v}), p_{e}^{K}(\boldsymbol{v})\right)$ be the solution of problem (2.2). Then, there exists $C>0$, independent of $h_{K}$, such that

$$
\begin{aligned}
\left|\boldsymbol{u}_{e}^{K}(\boldsymbol{v})\right|_{1, K} & \leq \lambda h_{K}\|\boldsymbol{v}\|_{0, K}, \\
\left\|p_{e}^{K}(\boldsymbol{v})\right\|_{0, K} & \leq C h_{K}\|\boldsymbol{v}\|_{0, K} .
\end{aligned}
$$

Moreover, there exists a constant $C>0$, independent of $h$, such that for all $q \in \mathbb{P}_{1}(K)$ there holds

$$
C h_{K}\|\nabla q\|_{0, K} \leq\left\|p_{e}^{K}(\nabla q)\right\|_{0, K} \leq h_{K}\|\nabla q\|_{0, K} .
$$

3.2. Existence and uniqueness of a discrete solution. We start defining the operator $\mathcal{P}: \boldsymbol{V}_{h} \longrightarrow Q_{h}$ by

$$
\begin{gathered}
\sum_{K \in \mathcal{T}_{h}} \alpha_{K}\left(p_{e}^{K}\left(\nabla \mathcal{P}\left(\boldsymbol{u}_{h}\right)\right), p_{e}^{K}\left(\nabla q_{h}\right)\right)_{K}+\sum_{F \in \mathcal{E}_{h}} \tilde{\tau}_{F}\left(\llbracket \mathcal{P}\left(\boldsymbol{u}_{h}\right) \boldsymbol{n} \rrbracket, \llbracket q_{h} \boldsymbol{n} \rrbracket\right)_{F} \\
=-\left(q_{h}, \nabla \cdot \boldsymbol{u}_{h}\right)-\sum_{K \in \mathcal{T}_{h}} \alpha_{K}\left(p_{e}^{K}\left(\lambda\left(\nabla \boldsymbol{u}_{h}\right) \boldsymbol{u}_{h}-\boldsymbol{f}\right), p_{e}^{K}\left(\nabla q_{h}\right)\right)_{K} \\
\quad-\sum_{F \in \mathcal{E}_{h}} \tilde{\tau}_{F}\left(\llbracket \partial_{\boldsymbol{n}} \boldsymbol{u}_{h} \rrbracket, \llbracket q_{h} \boldsymbol{n} \rrbracket\right)_{F}
\end{gathered}
$$

for all $\boldsymbol{u}_{h} \in \boldsymbol{V}_{h}, q_{h} \in Q_{h}$. The operator $\mathcal{P}$ is well defined thanks to the Lax-Milgram theorem with respect to the norm

$$
\left\|q_{h}\right\|_{*}:=\left\{\sum_{K \in \mathcal{T}_{h}} \alpha_{K}\left\|p_{e}^{K}\left(\nabla q_{h}\right)\right\|_{0, K}^{2}+\sum_{F \in \mathcal{E}_{h}} \tilde{\tau}_{F}\left\|\llbracket q_{h} \rrbracket\right\|_{0, F}^{2}\right\}^{1 / 2} .
$$

Also, we define the mapping $\mathcal{N}: \boldsymbol{V}_{h} \longrightarrow \boldsymbol{V}_{h}$ by

$$
\begin{aligned}
\left(\mathcal{N}\left(\boldsymbol{u}_{h}\right), \boldsymbol{v}_{h}\right) & \\
= & \left(\nabla \boldsymbol{u}_{h}, \nabla \boldsymbol{v}_{h}\right)+\left(\lambda\left(\nabla \boldsymbol{u}_{h}\right) \boldsymbol{u}_{h}, \boldsymbol{v}_{h}\right)-\left(\mathcal{P}\left(\boldsymbol{u}_{h}\right), \nabla \cdot \boldsymbol{v}_{h}\right)-\left(\boldsymbol{f}, \boldsymbol{v}_{h}\right) \\
& +\sum_{K \in \mathcal{T}_{h}} \gamma_{K}\left(\lambda \chi_{h}\left(\boldsymbol{x} \nabla \cdot \boldsymbol{u}_{h}\right), \lambda \chi_{h}\left(\boldsymbol{x} \nabla \cdot \boldsymbol{v}_{h}\right)\right)_{K} \\
& +\sum_{K \in \mathcal{T}_{h}} \alpha_{K}\left(p_{e}^{K}\left(\lambda\left(\nabla \boldsymbol{u}_{h}\right) \boldsymbol{u}_{h}-\boldsymbol{f}+\nabla \mathcal{P}\left(\boldsymbol{u}_{h}\right)\right), p_{e}^{K}\left(\lambda\left(\nabla \boldsymbol{v}_{h}\right) \boldsymbol{u}_{h}\right)\right)_{K} \\
& +\sum_{F \in \mathcal{E}_{h}} \tilde{\tau}_{F}\left(\llbracket \partial_{\boldsymbol{n}} \boldsymbol{u}_{h}+\mathcal{P}\left(\boldsymbol{u}_{h}\right) \boldsymbol{n} \rrbracket, \llbracket \partial_{\boldsymbol{n}} \boldsymbol{v}_{h} \rrbracket\right)_{F}
\end{aligned}
$$

for all $\boldsymbol{u}_{h}, \boldsymbol{v}_{h} \in \boldsymbol{V}_{h}$.

The following result provides a characterization of the solution of (3.4) in terms of the mappings $\mathcal{P}$ and $\mathcal{N}$.

Lemma 3.5. The pair $\left(\boldsymbol{u}_{h, \lambda}, p_{h, \lambda}\right) \in \boldsymbol{V}_{h} \times Q_{h}$ is a solution of problem (3.4) if and only if $\mathcal{N}\left(\boldsymbol{u}_{h, \lambda}\right)=\mathbf{0}$ and $p_{h, \lambda}=\mathcal{P}\left(\boldsymbol{u}_{h, \lambda}\right)$.

Proof. If $\mathcal{N}\left(\boldsymbol{u}_{h, \lambda}\right)=\mathbf{0}$ and $p_{h, \lambda}=\mathcal{P}\left(\boldsymbol{u}_{h, \lambda}\right)$, then adding (3.21) and (3.22) we see that $\left(\boldsymbol{u}_{h, \lambda}, p_{h, \lambda}\right) \in \boldsymbol{V}_{h} \times Q_{h}$ is a solution of problem (3.4). Moreover, let $\left(\boldsymbol{u}_{h, \lambda}, p_{h, \lambda}\right) \in$ $\boldsymbol{V}_{h} \times Q_{h}$ be a solution of (3.4). If $\boldsymbol{v}_{h}=\mathbf{0}$ in (3.4), then

$$
\begin{aligned}
& \sum_{K \in \mathcal{T}_{h}} \alpha_{K}\left(p_{e}^{K}\left(\nabla p_{h, \lambda}\right), p_{e}^{K}\left(\nabla q_{h}\right)\right)_{K}+\sum_{F \in \mathcal{E}_{h}} \tilde{\tau}_{F}\left(\llbracket p_{h, \lambda} \boldsymbol{n} \rrbracket, \llbracket q_{h} \boldsymbol{n} \rrbracket\right)_{F}=-\left(q_{h}, \nabla \cdot \boldsymbol{u}_{h, \lambda}\right) \\
& \quad-\sum_{K \in \mathcal{T}_{h}} \alpha_{K}\left(p_{e}^{K}\left(-\boldsymbol{f}+\lambda\left(\nabla \boldsymbol{u}_{h, \lambda}\right) \boldsymbol{u}_{h, \lambda}\right), p_{e}^{K}\left(\nabla q_{h}\right)\right)_{K}-\sum_{F \in \mathcal{E}_{h}} \tilde{\tau}_{F}\left(\llbracket \partial_{\boldsymbol{n}} \boldsymbol{u}_{h, \lambda} \rrbracket, \llbracket q_{h} \boldsymbol{n} \rrbracket\right)_{F}
\end{aligned}
$$

Copyright $@$ by SIAM. Unauthorized reproduction of this article is prohibited. 
and hence, since $\mathcal{P}$ is well defined, $p_{h, \lambda}=\mathcal{P}\left(\boldsymbol{u}_{h, \lambda}\right)$. Finally, if $q_{h}=0$ in (3.4), we have $\mathcal{N}\left(\boldsymbol{u}_{h, \lambda}\right)=\mathbf{0}$ and the result follows.

We are now in position to prove the existence and uniqueness of the solution of

THEOREM 3.6. There is a positive constant $\tilde{C}$, which is independent of $h$ and $\lambda$, such that problem (3.4) admits at least one solution $\left(\boldsymbol{u}_{h, \lambda}, p_{h, \lambda}\right)$ provided

$$
\lambda h^{1-\kappa}\left\{\|\boldsymbol{f}\|_{-1, \Omega}^{2}+\sum_{K \in \mathcal{T}_{h}} \alpha_{K}\left\|p_{e}^{K}(\boldsymbol{f})\right\|_{0, K}^{2}\right\}^{1 / 2} \leq \tilde{C},
$$

where $0<\kappa<1$. Moreover, the solution of problem (3.4) is unique provided $\lambda$ is sufficiently small. let

Proof. Let $R>0$ and $\boldsymbol{u}_{h} \in \boldsymbol{V}_{h}$ with $\left|\boldsymbol{u}_{h}\right|_{1, \Omega}=R$ be arbitrary and for abbreviation

$$
\begin{aligned}
& x:=\left\{\sum_{K \in \mathcal{T}_{h}} \alpha_{K}\left\|p_{e}^{K}\left(\lambda\left(\nabla \boldsymbol{u}_{h}\right) \boldsymbol{u}_{h}+\mathcal{P}\left(\boldsymbol{u}_{h}\right)\right)\right\|_{0, K}^{2}\right\}^{1 / 2}, \\
& y:=\left\{\sum_{F \in \mathcal{E}_{h}} \tilde{\tau}_{F}\left\|\llbracket \partial_{\boldsymbol{n}} \boldsymbol{u}_{h, \lambda}+\mathcal{P}\left(\boldsymbol{u}_{h}\right) \boldsymbol{n} \rrbracket\right\|_{0, F}^{2}\right\}^{1 / 2}, \\
& z:=\left\{\|\boldsymbol{f}\|_{-1, \Omega}^{2}+\sum_{K \in \mathcal{T}_{h}} \alpha_{K}\left\|p_{e}^{K}(\boldsymbol{f})\right\|_{0, K}^{2}\right\}^{1 / 2}, \\
& w:=\left\{\sum_{K \in \mathcal{T}_{h}} \gamma_{K}\left\|\lambda \chi_{h}\left(\boldsymbol{x} \nabla \cdot \boldsymbol{u}_{h}\right)\right\|_{0, K}^{2}\right\}^{1 / 2} .
\end{aligned}
$$

Taking $q_{h}=\mathcal{P}\left(\boldsymbol{u}_{h}\right)$ in (3.21) gives

$$
\begin{aligned}
-\left(\mathcal{P}\left(\boldsymbol{u}_{h}\right), \nabla \cdot \boldsymbol{u}_{h}\right)= & \sum_{K \in \mathcal{T}_{h}} \alpha_{K}\left(p_{e}^{K}\left(\lambda\left(\nabla \boldsymbol{u}_{h}\right) \boldsymbol{u}_{h}+\nabla \mathcal{P}\left(\boldsymbol{u}_{h}\right)-\boldsymbol{f}\right), p_{e}^{K}\left(\nabla \mathcal{P}\left(\boldsymbol{u}_{h}\right)\right)\right)_{K} \\
& +\sum_{F \in \mathcal{E}_{h}} \tilde{\tau}_{F}\left(\llbracket \partial_{\boldsymbol{n}} \boldsymbol{u}_{h}+\mathcal{P}\left(\boldsymbol{u}_{h}\right) \boldsymbol{n} \rrbracket, \llbracket \mathcal{P}\left(\boldsymbol{u}_{h}\right) \boldsymbol{n} \rrbracket\right)_{F} .
\end{aligned}
$$

Then, using Cauchy-Schwarz's inequality and (1.6) we have

$$
\begin{aligned}
\left(\mathcal{N}\left(\boldsymbol{u}_{h}\right), \boldsymbol{u}_{h}\right) & \\
= & \left|\boldsymbol{u}_{h}\right|_{1, \Omega}^{2}+\lambda\left(\left(\nabla \boldsymbol{u}_{h}\right) \boldsymbol{u}_{h}, \boldsymbol{u}_{h}\right)-\left(\boldsymbol{f}, \boldsymbol{u}_{h}\right) \\
& +\sum_{K \in \mathcal{T}_{h}} \alpha_{K}\left(p_{e}^{K}\left(\lambda\left(\nabla \boldsymbol{u}_{h}\right) \boldsymbol{u}_{h}+\nabla \mathcal{P}\left(\boldsymbol{u}_{h}\right)-\boldsymbol{f}\right), p_{e}^{K}\left(\lambda\left(\nabla \boldsymbol{u}_{h}\right) \boldsymbol{u}_{h}+\nabla \mathcal{P}\left(\boldsymbol{u}_{h}\right)\right)\right)_{K} \\
& +\sum_{K \in \mathcal{T}_{h}} \gamma_{K}\left\|\lambda \chi_{h}\left(\boldsymbol{x} \nabla \cdot \boldsymbol{u}_{h}\right)\right\|_{0, K}^{2}+\sum_{F \in \mathcal{E}_{h}} \tilde{\tau}_{F}\left\|\llbracket \partial_{\boldsymbol{n}} \boldsymbol{u}_{h}+\mathcal{P}\left(\boldsymbol{u}_{h}\right) \boldsymbol{n} \rrbracket\right\|_{0, F}^{2} \\
\geq & R^{2}+\lambda\left(\left(\nabla \boldsymbol{u}_{h}\right) \boldsymbol{u}_{h}, \boldsymbol{u}_{h}\right)-\|\boldsymbol{f}\|_{-1, \Omega}\left|\boldsymbol{u}_{h}\right|_{1, \Omega}+x^{2} \\
& -\sum_{K \in \mathcal{T}_{h}} \alpha_{K}\left(p_{e}^{K}(\boldsymbol{f}), p_{e}^{K}\left(\lambda\left(\nabla \boldsymbol{u}_{h}\right) \boldsymbol{u}_{h}+\nabla \mathcal{P}\left(\boldsymbol{u}_{h}\right)\right)\right)_{K}+w^{2}+y^{2} \\
\geq & \frac{1}{2} R^{2}+\frac{1}{2} x^{2}+y^{2}+w^{2}-\frac{1}{2} z^{2}-\frac{\lambda}{2}\left(\nabla \cdot \boldsymbol{u}_{h}, \boldsymbol{u}_{h} \cdot \boldsymbol{u}_{h}\right) .
\end{aligned}
$$

Copyright $\odot$ by SIAM. Unauthorized reproduction of this article is prohibited. 
Now, if we use the definition of the method with a test function given by $\left(\mathbf{0}, \mathcal{J}_{h}\left(\boldsymbol{u}_{h}\right.\right.$. $\left.\boldsymbol{u}_{h}\right)$ ), the Cauchy-Schwarz inequality, Lemma 3.4, (3.21), the fact that $\alpha_{K} \leq 1,(3.16)$, and the mesh regularity we get

$$
\begin{aligned}
& \left|\left(\nabla \cdot \boldsymbol{u}_{h}, \boldsymbol{u}_{h} \cdot \boldsymbol{u}_{h}\right)\right| \\
& \leq\left|\left(\nabla \cdot \boldsymbol{u}_{h}, \boldsymbol{u}_{h} \cdot \boldsymbol{u}_{h}-\mathcal{J}_{h}\left(\boldsymbol{u}_{h} \cdot \boldsymbol{u}_{h}\right)\right)\right|+\left|\left(\nabla \cdot \boldsymbol{u}_{h}, \mathcal{J}_{h}\left(\boldsymbol{u}_{h} \cdot \boldsymbol{u}_{h}\right)\right)\right| \\
& \leq \sqrt{2}\left|\boldsymbol{u}_{h}\right|_{1, \Omega}\left\|\boldsymbol{u}_{h} \cdot \boldsymbol{u}_{h}-\mathcal{J}_{h}\left(\boldsymbol{u}_{h} \cdot \boldsymbol{u}_{h}\right)\right\|_{0, \Omega} \\
& +\left|\sum_{K \in \mathcal{T}_{h}} \alpha_{K}\left(p_{e}^{K}\left(\lambda\left(\nabla \boldsymbol{u}_{h}\right) \boldsymbol{u}_{h}+\nabla \mathcal{P}\left(\boldsymbol{u}_{h}\right)-\boldsymbol{f}\right), p_{e}^{K}\left(\nabla \mathcal{J}_{h}\left(\boldsymbol{u}_{h} \cdot \boldsymbol{u}_{h}\right)\right)\right)_{K}\right| \\
& +\left|\sum_{F \in \mathcal{E}_{h}} \tilde{\tau}_{F}\left\|\llbracket \partial_{\boldsymbol{n}} \boldsymbol{u}_{h}+\mathcal{P}\left(\boldsymbol{u}_{h}\right) \boldsymbol{n} \rrbracket\right\|_{0, F}\left\|\llbracket \mathcal{J}_{h}\left(\boldsymbol{u}_{h} \cdot \boldsymbol{u}_{h}\right) \rrbracket\right\|_{0, F}\right| \\
& \leq C R\left\{\sum_{K \in \mathcal{T}_{h}} h_{K}^{2}\left|\boldsymbol{u}_{h} \cdot \boldsymbol{u}_{h}\right|_{1, K}^{2}\right\}^{1 / 2}+\sum_{K \in \mathcal{T}_{h}} \alpha_{K} \| p_{e}^{K}\left(\lambda\left(\nabla \boldsymbol{u}_{h}\right) \boldsymbol{u}_{h}\right. \\
& \left.+\nabla \mathcal{P}\left(\boldsymbol{u}_{h}\right)-\boldsymbol{f}\right)\left\|_{0, K}\right\| p_{e}^{K}\left(\nabla \mathcal{J}_{h}\left(\boldsymbol{u}_{h} \cdot \boldsymbol{u}_{h}\right)\right) \|_{0, K} \\
& +\sum_{F \in \mathcal{E}_{h}} \tilde{\tau}_{F}\left\|\llbracket \partial_{\boldsymbol{n}} \boldsymbol{u}_{h}+\mathcal{P}\left(\boldsymbol{u}_{h}\right) \boldsymbol{n} \rrbracket\right\|_{0, F}\left\|\llbracket \mathcal{J}_{h}\left(\boldsymbol{u}_{h} \cdot \boldsymbol{u}_{h}\right) \rrbracket\right\|_{0, F} \\
& \leq C R\left\{\sum_{K \in \mathcal{T}_{h}} h_{K}^{2}\left|\boldsymbol{u}_{h} \cdot \boldsymbol{u}_{h}\right|_{1, K}^{2}\right\}^{1 / 2}+C \sum_{K \in \mathcal{T}_{h}} \alpha_{K} \| p_{e}^{K}\left(\lambda\left(\nabla \boldsymbol{u}_{h}\right) \boldsymbol{u}_{h}\right. \\
& \left.+\nabla \mathcal{P}\left(\boldsymbol{u}_{h}\right)\right) \|_{0, K} h_{K}\left|\mathcal{J}_{h}\left(\boldsymbol{u}_{h} \cdot \boldsymbol{u}_{h}\right)\right|_{1, K} \\
& +C \sum_{K \in \mathcal{T}_{h}} \alpha_{K}\left\|p_{e}^{K}(\boldsymbol{f})\right\|_{0, K} h_{K}\left|J_{h}\left(\boldsymbol{u}_{h} \cdot \boldsymbol{u}_{h}\right)\right|_{1, K} \\
& +\sum_{F \in \mathcal{E}_{h}} \tilde{\tau}_{F}\left\|\llbracket \partial_{\boldsymbol{n}} \boldsymbol{u}_{h}+\mathcal{P}\left(\boldsymbol{u}_{h}\right) \boldsymbol{n} \rrbracket\right\|_{0, F}\left\|\llbracket \mathcal{J}_{h}\left(\boldsymbol{u}_{h} \cdot \boldsymbol{u}_{h}\right) \rrbracket\right\|_{0, F} \\
& \leq C R\left\{\sum_{K \in \mathcal{T}_{h}} h_{K}^{2}\left|\boldsymbol{u}_{h} \cdot \boldsymbol{u}_{h}\right|_{1, K}^{2}\right\}^{1 / 2}+C x\left\{\sum_{K \in \mathcal{T}_{h}} \alpha_{K} h_{K}^{2}\left|\boldsymbol{u}_{h} \cdot \boldsymbol{u}_{h}\right|_{1, \omega_{K}}^{2}\right\}^{1 / 2} \\
& +C\left\{\sum_{K \in \mathcal{T}_{h}} \alpha_{K}\left\|p_{e}^{K}(\boldsymbol{f})\right\|_{0, K}^{2}\right\}^{1 / 2}\left\{\sum_{K \in \mathcal{T}_{h}} \alpha_{K} h_{K}^{2}\left|\boldsymbol{u}_{h} \cdot \boldsymbol{u}_{h}\right|_{1, \omega_{K}}^{2}\right\}^{1 / 2} \\
& +\left\{\sum_{F \in \mathcal{E}_{h}} \tilde{\tau}_{F}\left\|\llbracket \partial_{\boldsymbol{n}} \boldsymbol{u}_{h}+\mathcal{P}\left(\boldsymbol{u}_{h}\right) \boldsymbol{n} \rrbracket\right\|_{0, F}^{2}\right\}^{1 / 2}\left\{\sum_{F \in \mathcal{E}_{h}} \tilde{\tau}_{F}\left\|\llbracket \mathcal{J}_{h}\left(\boldsymbol{u}_{h} \cdot \boldsymbol{u}_{h}\right) \rrbracket\right\|_{0, F}^{2}\right\}^{1 / 2} \\
& \leq C\{R+x+y+z\}\left\{\sum_{K \in \mathcal{T}_{h}} h_{K}^{2}\left|\boldsymbol{u}_{h} \cdot \boldsymbol{u}_{h}\right|_{1, K}^{2}+\sum_{F \in \mathcal{E}_{h}} \tilde{\tau}_{F}\left\|\llbracket \mathcal{J}_{h}\left(\boldsymbol{u}_{h} \cdot \boldsymbol{u}_{h}\right)-\boldsymbol{u}_{h} \cdot \boldsymbol{u}_{h} \rrbracket\right\|_{0, F}^{2}\right\}^{1 / 2} .
\end{aligned}
$$

But, in [2, Lemma 2], it is proved that

$$
\tilde{\tau}_{F}=\frac{1}{\lambda} \tau_{F} \leq C \nu \frac{h_{F}}{\nu}=C h_{F},
$$

Copyright (c) by SIAM. Unauthorized reproduction of this article is prohibited. 
and then, applying (3.17) and the mesh regularity, we arrive at

$$
\left|\left(\nabla \cdot \boldsymbol{u}_{h}, \boldsymbol{u}_{h} \cdot \boldsymbol{u}_{h}\right)\right| \leq C\{R+x+y+z\}\left\{\sum_{K \in \mathcal{T}_{h}} h_{K}^{2}\left|\boldsymbol{u}_{h} \cdot \boldsymbol{u}_{h}\right|_{1, K}^{2}\right\}^{1 / 2} .
$$

Moreover, using the local inverse inequality $\left\|v_{h}\right\|_{\infty, K} \leq C h_{K}^{-\frac{2}{q}}\left\|v_{h}\right\|_{0, q, K}$ for all $1 \leq$ $q \leq \infty$ (cf. [12]) and the Sobolev embedding theorem we obtain

$$
\begin{aligned}
\left|\boldsymbol{u}_{h} \cdot \boldsymbol{u}_{h}\right|_{1, K} & =\left\|\nabla\left(\boldsymbol{u}_{h} \cdot \boldsymbol{u}_{h}\right)\right\|_{0, K}=2\left\|\nabla\left(\boldsymbol{u}_{h}\right) \boldsymbol{u}_{h}\right\|_{0, K} \leq C\left|\boldsymbol{u}_{h}\right|_{1, K}\left\|\boldsymbol{u}_{h}\right\|_{\infty, K} \\
& \leq C h_{K}^{-\frac{2}{q}}\left|\boldsymbol{u}_{h}\right|_{1, K}\left\|\boldsymbol{u}_{h}\right\|_{q, K} \leq C h_{K}^{-\frac{2}{q}}\left|\boldsymbol{u}_{h}\right|_{1, K}\left\|\boldsymbol{u}_{h}\right\|_{q, \Omega} \leq C h_{K}^{-\frac{2}{q}}\left|\boldsymbol{u}_{h}\right|_{1, K}\left|\boldsymbol{u}_{h}\right|_{1, \Omega},
\end{aligned}
$$

then from (3.25) and (3.26), denoting $\kappa=\frac{2}{q}$, we arrive at

$$
\begin{aligned}
\left(\mathcal{N}\left(\boldsymbol{u}_{h}\right), \boldsymbol{u}_{h}\right) \geq & \frac{1}{2} R^{2}+\frac{1}{2} x^{2}+w^{2}+y^{2}-\frac{1}{2} z^{2}-\frac{\lambda}{2}\left(\nabla \cdot \boldsymbol{u}_{h}, \boldsymbol{u}_{h} \cdot \boldsymbol{u}_{h}\right) \\
\geq & \frac{1}{2} R^{2}+\frac{1}{2} x^{2}+w^{2}+y^{2}-\frac{1}{2} z^{2} \\
& -C \frac{\lambda}{2}\{R+x+y+z\}\left\{\sum_{K \in \mathcal{T}_{h}} h_{K}^{2-2 \kappa}\left|\boldsymbol{u}_{h}\right|_{1, K}^{2}\right\}^{1 / 2}\left|\boldsymbol{u}_{h}\right|_{1, \Omega} \\
\geq & \frac{1}{2} R^{2}+\frac{1}{2} x^{2}+w^{2}+y^{2}-\frac{1}{2} z^{2}-C h^{1-\kappa} \lambda\{R+x+y+z\} R^{2} \\
\geq & \frac{1}{2} R^{2}+\frac{1}{2} x^{2}+w^{2}+y^{2}-\frac{1}{2} z^{2}-C h^{1-\kappa} \lambda R^{3}-C h^{1-\kappa} \lambda\{x+y+z\} R^{2} \\
\geq & \frac{1}{2} R^{2}+\frac{1}{2} x^{2}+w^{2}+y^{2}-\frac{1}{2} z^{2} \\
& -C h^{1-\kappa} \lambda R^{3}-\frac{1}{2} x^{2}-\frac{1}{2} y^{2}-\frac{1}{2} z^{2}-\frac{3}{2} C^{2} h^{2(1-\kappa)} \lambda^{2} R^{4} \\
\geq & \frac{1}{2} R^{2}+w^{2}+\frac{1}{2} y^{2}-z^{2}-C h^{1-\kappa} \lambda R^{3}-\frac{3}{2} C^{2} h^{2(1-\kappa)} \lambda^{2} R^{4} .
\end{aligned}
$$

Let us define $R:=\frac{1}{M C \lambda h^{1-\kappa}}$ for an integer $M \geq 6$. It is clear that $M$ satisfies

$$
\frac{1}{2}-\frac{1}{M}-\frac{3}{2 M^{2}} \geq \frac{1}{4}
$$

Now, select $\tilde{C}=\frac{1}{2 M C}$ in (3.23) and observe that the assumption (3.23) leads to

$$
2 M C \lambda h^{1-\kappa} z \leq 1
$$

and we conclude from the definition of $R$ above that $z \leq \frac{R}{2}$. Gathering previous inequalities together, it holds that

$$
\begin{aligned}
\left(\mathcal{N}\left(\boldsymbol{u}_{h}\right), \boldsymbol{u}_{h}\right) & \geq\left(\frac{1}{2}-\frac{1}{M}-\frac{3}{2 M^{2}}\right) R^{2}-z^{2}+\frac{1}{2} y^{2}+w^{2} \\
& \geq \frac{1}{4} R^{2}-z^{2}+\frac{1}{2} y^{2}+w^{2} \\
& \geq \frac{1}{2} y^{2}+w^{2} \geq 0 .
\end{aligned}
$$

Copyright $\odot$ by SIAM. Unauthorized reproduction of this article is prohibited. 
Hence, Brouwer's fixed point theorem implies that there is a $\boldsymbol{u}_{h} \in \boldsymbol{V}_{h}$ with $\left|\boldsymbol{u}_{h}\right|_{1, \Omega} \leq$ $R$ and $\mathcal{N}\left(\boldsymbol{u}_{h}\right)=\mathbf{0}$. The uniqueness of solution follows from Banach's fixed point theorem using the arguments presented in [17] for standard finite elements. (See Appendix B for details.)

Remark 2.

- It is worth mentioning that the condition (3.23) can be avoided if the term $\left(\left(\nabla \boldsymbol{u}_{h}\right) \boldsymbol{u}_{h}, \boldsymbol{v}_{h}\right)$ in the RELP method is replaced by its antisymmetric counterpart $\frac{1}{2}\left[\left(\left(\nabla \boldsymbol{u}_{h}\right) \boldsymbol{u}_{h}, \boldsymbol{v}_{h}\right)-\left(\left(\nabla \boldsymbol{v}_{h}\right) \boldsymbol{u}_{h}, \boldsymbol{u}_{h}\right)\right]$. In fact, if $R$ is set larger than $\sqrt{2} z$, then (3.25) becomes positive as the last term in (3.25) is absent. Also, the error estimate given in the next section remains valid for such a case.

- Observe that the properties of $p_{e}^{K}$ used to prove Theorem 3.6 were linearity and the stability result given in Lemma 3.4. Thereby, the exact same proof applies to the simplified method (2.4) by replacing $p_{e}^{K}$ by $p_{e}^{K} \circ \Pi_{K}$ and using the stability of $\Pi_{K}$ given in (3.9).

- For the three-dimensional case, the same argument used in Theorem 3.6 can be applied, but in this case the Sobolev embedding theorem only ensures $\left\|\boldsymbol{u}_{h}\right\|_{q, \Omega} \leq C\left|\boldsymbol{u}_{h}\right|_{1, \Omega}$ for $1 \leq q \leq 6$, and then $\kappa$ must be supposed to be smaller than $\frac{1}{3}$. Also, the proof of uniqueness detailed in Appendix B holds in the three-dimensional setting with minor changes.

- Theorem 3.6 is also valid for the case in which the method is applied with elements of higher order. The only modification needed in the proof is to change the definition of $x$ in (3.24) to $x:=\left\{\sum_{K \in \mathcal{T}_{h}} \alpha_{K} \| p_{e}^{K}\left(-\Delta \boldsymbol{u}_{h}+\lambda\left(\nabla \boldsymbol{u}_{h}\right) \boldsymbol{u}_{h}+\right.\right.$ $\left.\left.\mathcal{P}\left(\boldsymbol{u}_{h}\right)\right) \|_{0, K}^{2}\right\}^{1 / 2}$.

3.3. A priori error analysis. We place the method in the case that wellposedness is ensured, i.e., at the diffusion dominated regime. To this end, we suppose that $\alpha_{K}=\gamma_{K}=1$ on every element. On each edge we take $\tau_{F}=\frac{h_{F}}{12}$ since, in the diffusion dominated case, both expressions are equivalent. (For details see Lemma 2 in [2].) Then, method (3.4) simplifies to the following: Find $\left(\boldsymbol{u}_{h, \lambda}, p_{h, \lambda}\right) \in \boldsymbol{V}_{h} \times Q_{h}$ such that

$$
\begin{aligned}
\left(\nabla \boldsymbol{u}_{h, \lambda}, \nabla \boldsymbol{v}_{h}\right)+\lambda\left(\left(\nabla \boldsymbol{u}_{h, \lambda}\right) \boldsymbol{u}_{h, \lambda}, \boldsymbol{v}_{h}\right)-\left(p_{h, \lambda}, \nabla \cdot \boldsymbol{v}_{h}\right)+\left(q_{h}, \nabla \cdot \boldsymbol{u}_{h, \lambda}\right) & \quad-\sum_{K \in \mathcal{T}_{h}}\left(p_{e}^{K}\left(\boldsymbol{f}+\Delta \boldsymbol{u}_{h, \lambda}-\lambda\left(\nabla \boldsymbol{u}_{h, \lambda}\right) \boldsymbol{u}_{h, \lambda}-\nabla p_{h, \lambda}\right),\right. \\
& \left.p_{e}^{K}\left(\nabla q_{h}+\lambda\left(\nabla \boldsymbol{v}_{h}\right) \boldsymbol{u}_{h, \lambda}\right)\right)_{K} \\
& +\sum_{K \in \mathcal{T}_{h}}\left(\lambda \chi_{h}\left(\boldsymbol{x} \nabla \cdot \boldsymbol{u}_{h, \lambda}\right), \lambda \chi_{h}\left(\boldsymbol{x} \nabla \cdot \boldsymbol{v}_{h}\right)\right)_{K} \\
& +\sum_{F \in \mathcal{E}_{h}} \frac{h_{F}}{12}\left(\llbracket \partial_{\boldsymbol{n}} \boldsymbol{u}_{h, \lambda}+p_{h, \lambda} \boldsymbol{n} \rrbracket, \llbracket \partial_{\boldsymbol{n}} \boldsymbol{v}_{h}+q_{h} \boldsymbol{n} \rrbracket\right)_{F} \\
= & \left(\boldsymbol{f}, \boldsymbol{v}_{h}\right) \quad \forall\left(\boldsymbol{v}_{h}, q_{h}\right) \in \boldsymbol{V}_{h} \times Q_{h} .
\end{aligned}
$$

Next, we denote by $T_{h}: \boldsymbol{V}^{\prime} \times Q \longrightarrow \boldsymbol{V}_{h} \times Q_{h}$ the discrete Stokes operator, which associates with each $(\boldsymbol{w}, r) \in \boldsymbol{V}^{\prime} \times Q$ the unique solution $\left(\boldsymbol{u}_{h}, p_{h}\right) \in \boldsymbol{V}_{h} \times Q_{h}$ of

$$
\begin{array}{r}
\left(\nabla \boldsymbol{u}_{h}, \nabla \boldsymbol{v}_{h}\right)-\left(p_{h}, \nabla \cdot \boldsymbol{v}_{h}\right)+\left(q_{h}, \nabla \cdot \boldsymbol{u}_{h}\right)+\sum_{K \in \mathcal{T}_{h}}\left(p_{e}^{K}\left(\nabla p_{h}\right), p_{e}^{K}\left(\nabla q_{h}\right)\right)_{K} \\
+\left(\lambda \chi_{h}\left(\boldsymbol{x} \nabla \cdot \boldsymbol{u}_{h}\right), \lambda \chi_{h}\left(\boldsymbol{x} \nabla \cdot \boldsymbol{v}_{h}\right)\right)_{K}+\sum_{F \in \mathcal{E}_{h}} \frac{h_{F}}{12}\left(\llbracket \partial_{\boldsymbol{n}} \boldsymbol{u}_{h}+p_{h} \boldsymbol{n} \rrbracket, \llbracket \partial_{\boldsymbol{n}} \boldsymbol{v}_{h}+q_{h} \boldsymbol{n} \rrbracket\right)_{F} \\
=\left\langle\boldsymbol{w}, \boldsymbol{v}_{h}\right\rangle+\left(r, q_{h}\right)
\end{array}
$$

Copyright $@$ by SIAM. Unauthorized reproduction of this article is prohibited. 
for all $\left(\boldsymbol{v}_{h}, q_{h}\right) \in \boldsymbol{V}_{h} \times Q_{h}$. This discrete Stokes operator, to the best of our knowledge, does not coincide with any stabilized method available in the literature, although it might be seen as a variation of the PPS method (cf. [11]) if the pressure space is the continuous piecewise linear functions or the RELP method (cf. [1]) if the pressure is discontinuous. Adapting the analysis presented in $[5,1]$ we obtain the following result. (See Appendix A for a sketch of the analysis.)

Lemma 3.7. There exist constants $C, C^{\prime}>0$, independent of $h$ and $\lambda$, such that the following holds:

$$
\begin{aligned}
\left\|\left(T-T_{h}\right)(\boldsymbol{w}, 0)\right\| & \leq C h(1+\lambda h)^{2}\|\boldsymbol{w}\|_{0, \Omega} \quad \forall \boldsymbol{w} \in L^{2}(\Omega), \\
\left\|T_{h}(\boldsymbol{w}, q)\right\| & \leq C^{\prime}(1+\lambda h)^{2}\|(\boldsymbol{w}, q)\|_{\left(\boldsymbol{V}_{h} \times Q_{h}\right)^{\prime}} \quad \forall(\boldsymbol{w}, q) \in(\boldsymbol{V} \times Q)^{\prime} .
\end{aligned}
$$

To write the discrete method as a fixed point equation we also introduce the mapping $G_{h}: \Lambda \times H^{2}\left(\mathcal{T}_{h}\right)^{2} \times H^{1}\left(\mathcal{T}_{h}\right) \longrightarrow \boldsymbol{V}_{h} \times Q_{h}$ by $G_{h}(\lambda, \boldsymbol{z}, t)=\left(\boldsymbol{w}_{h}, r_{h}\right)$, where

$$
\begin{aligned}
\left(\boldsymbol{w}_{h}, \boldsymbol{v}_{h}\right)+\left(r_{h}, q_{h}\right)= & -\left(\boldsymbol{f}-\lambda(\nabla \boldsymbol{z}) \boldsymbol{z}, \boldsymbol{v}_{h}\right)-\sum_{K \in \mathcal{T}_{h}}\left(p_{e}^{K}(\boldsymbol{f}-\lambda(\nabla \boldsymbol{z}) \boldsymbol{z}), p_{e}^{K}\left(\nabla q_{h}\right)\right)_{K} \\
& -\sum_{K \in \mathcal{T}_{h}}\left(p_{e}^{K}(\boldsymbol{f}+\Delta \boldsymbol{z}-\lambda(\nabla \boldsymbol{z}) \boldsymbol{z}-\nabla t), p_{e}^{K}\left(\lambda\left(\nabla \boldsymbol{v}_{h}\right) \boldsymbol{z}\right)\right)_{K}
\end{aligned}
$$

for all $\left(\boldsymbol{v}_{h}, q_{h}\right) \in \boldsymbol{V}_{h} \times Q_{h}$.

Using these operators, problem (3.27) can be written in a form analogous to (3.3):

$$
F_{h}\left(\lambda, \boldsymbol{u}_{h, \lambda}, p_{h, \lambda}\right):=\left(\boldsymbol{u}_{h, \lambda}, p_{h, \lambda}\right)+T_{h} G_{h}\left(\lambda, \boldsymbol{u}_{h, \lambda}, p_{h, \lambda}\right)=\mathbf{0} .
$$

The next result states some properties of $F_{h}$ and its derivatives and will be fundamental for the error analysis carried out below. The first one proves that the differential operator $D_{\boldsymbol{u}, p} F_{h}\left(\lambda, \mathcal{I}_{h} \boldsymbol{u}_{\lambda}, \mathcal{J}_{h} p_{\lambda}\right)$ is an isomorphism under appropriate conditions on $h$ and $\lambda$.

Lemma 3.8. Assume that on a given compact interval $\Lambda \subset \mathbb{R}$ a regular branch $\lambda \rightarrow\left(\boldsymbol{u}_{\lambda}, p_{\lambda}\right)$ of solutions of problem (3.3) exists and that $\left(\boldsymbol{u}_{\lambda}, p_{\lambda}\right)$ belongs to the space $H^{2}(\Omega)^{2} \times H^{1}(\Omega)$. Therefore, there exists a constant $h_{0}>0$ such that for all $h \leq h_{0}$, the mapping $D_{\boldsymbol{u}, p} F_{h}\left(\lambda, \mathcal{I}_{h} \boldsymbol{u}_{\lambda}, \mathcal{J}_{h} p_{\lambda}\right)$ is an isomorphism on $\boldsymbol{V}_{h} \times Q_{h}$.

Proof. We start noting that since $T$ is a linear and continuous operator, and using (1.5) and (3.11), we arrive at

$$
\begin{aligned}
& \left\|D_{\boldsymbol{u}, p} F\left(\lambda, \boldsymbol{u}_{\lambda}, p_{\lambda}\right)-D_{\boldsymbol{u}, p} F\left(\lambda, \mathcal{I}_{h} \boldsymbol{u}_{\lambda}, \mathcal{J}_{h} p_{\lambda}\right)\right\|_{\mathcal{L}(\boldsymbol{V} \times Q)} \\
& \quad=\sup _{\|(\boldsymbol{v}, q)\| \leq 1}\left\|T\left(\left(D_{\boldsymbol{u}, p} G\left(\lambda, \boldsymbol{u}_{\lambda}, p_{\lambda}\right)-D_{\boldsymbol{u}, p} G\left(\lambda, \mathcal{I}_{h} \boldsymbol{u}_{\lambda}, \mathcal{J}_{h} p_{\lambda}\right)\right)[\boldsymbol{v}, q]\right)\right\| \\
& \quad \leq C \sup _{\|(\boldsymbol{v}, q)\| \leq 1}\left\|\left(D_{\boldsymbol{u}, p} G\left(\lambda, \boldsymbol{u}_{\lambda}, p_{\lambda}\right)-D_{\boldsymbol{u}, p} G\left(\lambda, \mathcal{I}_{h} \boldsymbol{u}_{\lambda}, \mathcal{J}_{h} p_{\lambda}\right)\right)[\boldsymbol{v}, q]\right\|_{(\boldsymbol{V} \times Q)^{\prime}} \\
& \quad \leq C \lambda \sup _{\|(\boldsymbol{w}, t)\| \leq 1} \sup _{\|(\boldsymbol{v}, q)\| \leq 1}\left(\left(\nabla \boldsymbol{u}_{\lambda}\right) \boldsymbol{v}+(\nabla \boldsymbol{v}) \boldsymbol{u}_{\lambda}-\left(\nabla \mathcal{I}_{h} \boldsymbol{u}_{\lambda}\right) \boldsymbol{v}-(\nabla \boldsymbol{v}) \mathcal{I}_{h} \boldsymbol{u}_{\lambda}, \boldsymbol{w}\right) \\
& \quad=C \lambda \sup _{\|(\boldsymbol{w}, t)\| \leq 1} \sup _{\|(\boldsymbol{v}, q)\| \leq 1}\left(\left(\nabla\left(\boldsymbol{u}_{\lambda}-\mathcal{I}_{h} \boldsymbol{u}_{\lambda}\right)\right) \boldsymbol{v}+(\nabla \boldsymbol{v})\left(\boldsymbol{u}_{\lambda}-\mathcal{I}_{h} \boldsymbol{u}_{\lambda}\right), \boldsymbol{w}\right) \\
& \quad \leq C \lambda \sup _{\|(\boldsymbol{w}, t)\| \leq 1} \sup _{\|(\boldsymbol{v}, q)\| \leq 1} 2\left|\boldsymbol{u}_{\lambda}-\mathcal{I}_{h} \boldsymbol{u}_{\lambda}\right|_{1, \Omega}|\boldsymbol{v}|_{1, \Omega}|\boldsymbol{w}|_{1, \Omega} \\
& \quad \leq C \lambda h L,
\end{aligned}
$$

where $L:=\sup _{\lambda \in \Lambda} \max \left\{\|\boldsymbol{f}\|_{0, \Omega},\left\|\boldsymbol{u}_{\lambda}\right\|_{2, \Omega},\left\|p_{\lambda}\right\|_{1, \Omega}\right\}$. Then, since the set of isomorphisms on $\boldsymbol{V} \times Q$ is open, there exists $h_{2}>0$ such that $D_{\boldsymbol{u}, p} F\left(\lambda, \mathcal{I}_{h} \boldsymbol{u}_{\lambda}, \mathcal{J}_{h} p_{\lambda}\right)$ is an isomorphism on $\boldsymbol{V} \times Q$ for all $h \leq h_{2}$.

Copyright (c) by SIAM. Unauthorized reproduction of this article is prohibited. 
Next, we define the mapping $A_{1}:=I+T_{h} D_{\boldsymbol{u}, p} G\left(\lambda, \mathcal{I}_{h} \boldsymbol{u}_{\lambda}, \mathcal{J}_{h} p_{\lambda}\right)$, which belongs to $\mathcal{L}(\boldsymbol{V} \times Q)$ but also to $\mathcal{L}\left(\boldsymbol{V}_{h} \times Q_{h}\right)$. Then, we use Lemma 3.7, Hölder's inequality, (3.14), (3.15), and the inclusions $H^{2}(\Omega) \hookrightarrow L^{\infty}(\Omega)$ and $H^{1}(\Omega) \hookrightarrow L^{4}(\Omega)$, to obtain

$$
\begin{aligned}
& \left\|A_{1}-D_{\boldsymbol{u}, p} F\left(\lambda, \mathcal{I}_{h} \boldsymbol{u}_{\lambda}, \mathcal{J}_{h} p_{\lambda}\right)\right\|_{\mathcal{L}(\boldsymbol{V} \times Q)} \\
& =\sup _{\|(\boldsymbol{v}, q)\| \leq 1}\left\|\left(T_{h}-T\right) D_{\boldsymbol{u}, p} G\left(\lambda, \mathcal{I}_{h} \boldsymbol{u}_{\lambda}, \mathcal{J}_{h} p_{\lambda}\right)[\boldsymbol{v}, q]\right\| \\
& \leq C(1+\lambda h)^{2} \lambda h \sup _{\|\boldsymbol{w}\|_{0, \Omega} \leq 1} \sup _{\|(\boldsymbol{v}, q)\| \leq 1}\left(\left(\nabla \mathcal{I}_{h} \boldsymbol{u}_{\lambda}\right) \boldsymbol{v}+(\nabla \boldsymbol{v}) \mathcal{I}_{h} \boldsymbol{u}_{\lambda}, \boldsymbol{w}\right) \\
& \leq C(1+\lambda h)^{2} \lambda h \sup _{\|\boldsymbol{w}\|_{0, \Omega} \leq 1} \sup _{\|(\boldsymbol{v}, q)\| \leq 1}\left\{\left\|\nabla \mathcal{I}_{h} \boldsymbol{u}_{\lambda}\right\|_{0,4, \Omega}\|\boldsymbol{v}\|_{0,4, \Omega}\|\boldsymbol{w}\|_{0, \Omega}\right. \\
& \left.+\|\nabla \boldsymbol{v}\|_{0, \Omega}\left\|\mathcal{I}_{h} \boldsymbol{u}_{\lambda}\right\|_{\infty, \Omega}\|\boldsymbol{w}\|_{0, \Omega}\right\} \\
& \leq C(1+\lambda h)^{2} \lambda h \sup _{\|\boldsymbol{w}\|_{0, \Omega} \leq 1} \sup _{\|(\boldsymbol{v}, q)\| \leq 1}\left\{\left\|\nabla \boldsymbol{u}_{\lambda}\right\|_{0,4, \Omega}\|\boldsymbol{v}\|_{0,4, \Omega}\|\boldsymbol{w}\|_{0, \Omega}\right. \\
& \left.+\left\|\boldsymbol{u}_{\lambda}\right\|_{\infty, \Omega}\|\nabla \boldsymbol{v}\|_{0, \Omega}\|\boldsymbol{w}\|_{0, \Omega}\right\} \\
& \leq C(1+\lambda h)^{2} \lambda h \sup _{\|\boldsymbol{w}\|_{0, \Omega} \leq 1} \sup _{\|(\boldsymbol{v}, q)\| \leq 1}\left\{\left\|\boldsymbol{u}_{\lambda}\right\|_{2, \Omega}|\boldsymbol{v}|_{1, \Omega}\|\boldsymbol{w}\|_{0, \Omega}+\left\|\boldsymbol{u}_{\lambda}\right\|_{2, \Omega}|\boldsymbol{v}|_{1, \Omega}\|\boldsymbol{w}\|_{0, \Omega}\right\} \\
& \leq C(1+\lambda h)^{2} \lambda h L,
\end{aligned}
$$

and then there exists $h_{1} \leq h_{2}$ such that for all $h \leq h_{1}$ the mapping $A_{1}$ is an isomorphism in $\boldsymbol{V} \times Q$. Also, since $A_{1}$ also maps $\boldsymbol{V}_{h} \times Q_{h}$ onto itself and is injective, it is also an isomorphism on $\boldsymbol{V}_{h} \times Q_{h}$.

Finally, using Lemma 3.7, it holds that

$$
\begin{aligned}
& \left\|A_{1}-D_{\boldsymbol{u}, p} F_{h}\left(\lambda, \mathcal{I}_{h} \boldsymbol{u}_{\lambda}, \mathcal{J}_{h} p_{\lambda}\right)\right\|_{\mathcal{L}\left(\boldsymbol{V}_{h} \times Q_{h}\right)} \\
& =\sup _{\left\|\left(\boldsymbol{v}_{h}, q_{h}\right)\right\| \leq 1}\left\|T_{h}\left(D_{\boldsymbol{u}, p} G_{h}\left(\lambda, \mathcal{I}_{h} \boldsymbol{u}_{\lambda}, \mathcal{J}_{h} p_{\lambda}\right)-D_{\boldsymbol{u}, p} G\left(\lambda, \mathcal{I}_{h} \boldsymbol{u}_{\lambda}, \mathcal{J}_{h} p_{\lambda}\right)\right)\left[\boldsymbol{v}_{h}, q_{h}\right]\right\| \\
& \leq C(1+\lambda h)^{2} \sup _{\left\|\left(\boldsymbol{v}_{h}, q_{h}\right)\right\| \leq 1} \|\left(D_{\boldsymbol{u}, p} G_{h}\left(\lambda, \mathcal{I}_{h} \boldsymbol{u}_{\lambda}, \mathcal{J}_{h} p_{\lambda}\right)\right. \\
& \left.-D_{\boldsymbol{u}, p} G\left(\lambda, \mathcal{I}_{h} \boldsymbol{u}_{\lambda}, \mathcal{J}_{h} p_{\lambda}\right)\right)\left[\boldsymbol{v}_{h}, q_{h}\right] \|_{\left(\boldsymbol{V}_{h} \times Q_{h}\right)^{\prime}} \\
& \leq C(1+\lambda h)^{2} \sup _{\left\|\left(\boldsymbol{w}_{h}, t_{h}\right)\right\| \leq 1} \sup _{\left\|\left(\boldsymbol{v}_{h}, q_{h}\right)\right\| \leq 1} \\
& \left\{\sum_{K \in \mathcal{T}_{h}}\left(p_{e}^{K}\left(\boldsymbol{f}-\lambda\left(\nabla \mathcal{I}_{h} \boldsymbol{u}_{\lambda}\right) \mathcal{I}_{h} \boldsymbol{u}_{\lambda}-\nabla \mathcal{J}_{h} p_{\lambda}\right), p_{e}^{K}\left(\lambda\left(\nabla \boldsymbol{w}_{h}\right) \boldsymbol{v}_{h}\right)\right)_{K}\right. \\
& -\sum_{K \in \mathcal{T}_{h}}\left(p_{e}^{K}\left(\lambda\left(\nabla \boldsymbol{v}_{h}\right) \mathcal{I}_{h} \boldsymbol{u}_{\lambda}+\lambda\left(\nabla \mathcal{I}_{h} \boldsymbol{u}_{\lambda}\right) \boldsymbol{v}_{h}+\nabla q_{h}\right), p_{e}^{K}\left(\lambda\left(\nabla \boldsymbol{w}_{h}\right) \mathcal{I}_{h} \boldsymbol{u}_{\lambda}\right)\right)_{K} \\
& \left.-\sum_{K \in \mathcal{T}_{h}}\left(p_{e}^{K}\left(\lambda\left(\nabla \boldsymbol{v}_{h}\right) \mathcal{I}_{h} \boldsymbol{u}_{\lambda}+\lambda\left(\nabla \mathcal{I}_{h} \boldsymbol{u}_{\lambda}\right) \boldsymbol{v}_{h}\right), p_{e}^{K}\left(\nabla t_{h}\right)\right)_{K}\right\} \\
& =C(1+\lambda h)^{2} \sup _{\left\|\left(\boldsymbol{w}_{h}, t_{h}\right)\right\| \leq 1} \sup _{\left\|\left(\boldsymbol{v}_{h}, q_{h}\right)\right\| \leq 1}\{\mathrm{I}+\mathrm{II}+\mathrm{III}\} .
\end{aligned}
$$

Now, using (3.5), (3.11), (3.12), and the embedding $H^{2}(\Omega) \hookrightarrow L^{\infty}(\Omega)$, we obtain

$$
\begin{aligned}
& \left\|\left(\nabla \boldsymbol{u}_{\lambda}\right) \boldsymbol{u}_{\lambda}-\left(\nabla \mathcal{I}_{h} \boldsymbol{u}_{\lambda}\right) \mathcal{I}_{h} \boldsymbol{u}_{\lambda}\right\|_{0, K} \\
& \quad \leq\left\|\left(\nabla\left(\boldsymbol{u}_{\lambda}-\mathcal{I}_{h} \boldsymbol{u}_{\lambda}\right)\right) \boldsymbol{u}_{\lambda}\right\|_{0, K}+\left\|\left(\nabla \mathcal{I}_{h} \boldsymbol{u}_{\lambda}\right)\left(\boldsymbol{u}_{\lambda}-\mathcal{I}_{h} \boldsymbol{u}_{\lambda}\right)\right\|_{0, K} \\
& \quad \leq\left\|\boldsymbol{u}_{\lambda}\right\|_{\infty, K}\left|\boldsymbol{u}_{\lambda}-\mathcal{I}_{h} \boldsymbol{u}_{\lambda}\right|_{1, K}+\left\|\nabla \mathcal{I}_{h} \boldsymbol{u}_{\lambda}\right\|_{\infty, K}\left\|\boldsymbol{u}_{\lambda}-\mathcal{I}_{h} \boldsymbol{u}_{\lambda}\right\|_{0, K} \\
& \quad \leq\left\|\boldsymbol{u}_{\lambda}\right\|_{\infty, \Omega} C h_{K}\left\|\boldsymbol{u}_{\lambda}\right\|_{2, K}+C h_{K}^{-1}\left\|\nabla \mathcal{I}_{h} \boldsymbol{u}_{\lambda}\right\|_{0, K} h_{K}^{2}\left\|\boldsymbol{u}_{\lambda}\right\|_{2, K} \\
& \quad \leq C h_{K} L\left\|\boldsymbol{u}_{\lambda}\right\|_{2, K},
\end{aligned}
$$

Copyright (c) by SIAM. Unauthorized reproduction of this article is prohibited. 
and then, using (3.16), we get

$$
\begin{aligned}
\| & -\boldsymbol{f}+\lambda\left(\nabla \mathcal{I}_{h} \boldsymbol{u}_{\lambda}\right) \mathcal{I}_{h} \boldsymbol{u}_{\lambda}+\nabla \mathcal{J}_{h} p_{\lambda} \|_{0, K} \\
& =\left\|\Delta \boldsymbol{u}_{\lambda}-\lambda\left(\left(\nabla \boldsymbol{u}_{\lambda}\right) \boldsymbol{u}_{\lambda}-\left(\nabla \mathcal{I}_{h} \boldsymbol{u}_{\lambda}\right) \mathcal{I}_{h} \boldsymbol{u}_{\lambda}\right)-\nabla\left(p_{\lambda}-\mathcal{J}_{h} p_{\lambda}\right)\right\|_{0, K} \\
& \leq\left\|\Delta \boldsymbol{u}_{\lambda}\right\|_{0, K}+C \lambda h_{K} L\left\|\boldsymbol{u}_{\lambda}\right\|_{2, K}+C\left\|p_{\lambda}\right\|_{1, \omega_{K}} \\
& \leq C\left\{\left(1+\lambda h_{K} L\right)\left\|\boldsymbol{u}_{\lambda}\right\|_{2, K}+\left\|p_{\lambda}\right\|_{1, \omega_{K}}\right\} .
\end{aligned}
$$

Item I is addressed using Lemma 3.4, (3.30) and (3.5), and Poincaré's inequality as follows:

$$
\begin{aligned}
\mathrm{I} & =\sum_{K \in \mathcal{T}_{h}}\left(p_{e}^{K}\left(\boldsymbol{f}-\lambda\left(\nabla \mathcal{I}_{h} \boldsymbol{u}_{\lambda}\right) \mathcal{I}_{h} \boldsymbol{u}_{\lambda}-\nabla \mathcal{J}_{h} p_{\lambda}\right), p_{e}^{K}\left(\lambda\left(\nabla \boldsymbol{w}_{h}\right) \boldsymbol{v}_{h}\right)\right)_{K} \\
& \leq C \sum_{K \in \mathcal{T}_{h}} \lambda h_{K}^{2}\left\|\boldsymbol{f}-\lambda\left(\nabla \mathcal{I}_{h} \boldsymbol{u}_{\lambda}\right) \mathcal{I}_{h} \boldsymbol{u}_{\lambda}-\nabla \mathcal{J}_{h} p_{\lambda}\right\|_{0, K}\left\|\left(\nabla \boldsymbol{w}_{h}\right) \boldsymbol{v}_{h}\right\|_{0, K} \\
& \leq C \sum_{K \in \mathcal{T}_{h}} \lambda h_{K} L\left(1+\lambda h_{K} L\right)\left\|\nabla \boldsymbol{w}_{h}\right\|_{0, K} h_{K}\left\|\boldsymbol{v}_{h}\right\|_{\infty, K} \\
& \leq C \lambda h L(1+\lambda h L) \sum_{K \in \mathcal{T}_{h}}\left\|\nabla \boldsymbol{w}_{h}\right\|_{0, K}\left\|\boldsymbol{v}_{h}\right\|_{0, K} \\
& \leq C \lambda h L(1+\lambda h L)\left\|\left(\boldsymbol{w}_{h}, t_{h}\right)\right\|\left\|\left(\boldsymbol{v}_{h}, q_{h}\right)\right\| .
\end{aligned}
$$

Also, again using Lemma 3.4, the embedding of $H^{2}(\Omega) \hookrightarrow L^{\infty}(\Omega),(3.7),(3.14),(3.15)$, and the continuous embedding $H^{1}(\Omega) \hookrightarrow L^{4}(\Omega)$, item II is bounded as

$$
\begin{aligned}
& \mathrm{II}=-\sum_{K \in \mathcal{T}_{h}}\left(p_{e}^{K}\left(\lambda\left(\nabla \boldsymbol{v}_{h}\right) \mathcal{I}_{h} \boldsymbol{u}_{\lambda}+\lambda\left(\nabla \mathcal{I}_{h} \boldsymbol{u}_{\lambda}\right) \boldsymbol{v}_{h}+\nabla q_{h}\right), p_{e}^{K}\left(\lambda\left(\nabla \boldsymbol{w}_{h}\right) \mathcal{I}_{h} \boldsymbol{u}_{\lambda}\right)\right)_{K} \\
& \leq C \lambda \sum_{K \in \mathcal{T}_{h}}\left\{\lambda\left\|p_{e}^{K}\left(\left(\nabla \boldsymbol{v}_{h}\right) \mathcal{I}_{h} \boldsymbol{u}_{\lambda}+\left(\nabla \mathcal{I}_{h} \boldsymbol{u}_{\lambda}\right) \boldsymbol{v}_{h}\right)\right\|_{0, K}\right. \\
& \left.+\left\|p_{e}^{K}\left(\nabla q_{h}\right)\right\|_{0, K}\right\}\left\|p_{e}^{K}\left(\left(\nabla \boldsymbol{w}_{h}\right) \mathcal{I}_{h} \boldsymbol{u}_{\lambda}\right)\right\|_{0, K} \\
& \leq C \lambda \sum_{K \in \mathcal{T}_{h}}\left\{\lambda h_{K}\left(\left\|\left(\nabla \boldsymbol{v}_{h}\right) \mathcal{I}_{h} \boldsymbol{u}_{\lambda}\right\|_{0, K}+\left\|\left(\nabla \mathcal{I}_{h} \boldsymbol{u}_{\lambda}\right) \boldsymbol{v}_{h}\right\|_{0, K}\right)\right. \\
& \left.+h_{K}\left\|\nabla q_{h}\right\|_{0, K}\right\} h_{K}\left\|\left(\nabla \boldsymbol{w}_{h}\right) \mathcal{I}_{h} \boldsymbol{u}_{\lambda}\right\|_{0, K} \\
& \leq C \lambda h \sum_{K \in \mathcal{T}_{h}}\left\{\lambda h_{K}\left\|\nabla \boldsymbol{v}_{h}\right\|_{0, K}\left\|\mathcal{I}_{h} \boldsymbol{u}_{\lambda}\right\|_{\infty, K}+\lambda h_{K}\left\|\nabla \mathcal{I}_{h} \boldsymbol{u}_{\lambda}\right\|_{0,4, K}\left\|\boldsymbol{v}_{h}\right\|_{0,4, K}\right. \\
& \left.+h_{K}\left\|\nabla q_{h}\right\|_{0, K}\right\}\left\|\nabla \boldsymbol{w}_{h}\right\|_{0, K}\left\|\mathcal{I}_{h} \boldsymbol{u}_{\lambda}\right\|_{\infty, K} \\
& \leq C \lambda h L\left\{\lambda h\left\|\nabla \boldsymbol{v}_{h}\right\|_{0, \Omega}\left\|\boldsymbol{u}_{\lambda}\right\|_{\infty, \Omega}+\lambda h\left\|\nabla \boldsymbol{u}_{\lambda}\right\|_{0,4, \Omega}\left\|\boldsymbol{v}_{h}\right\|_{0,4, \Omega}+\left\|q_{h}\right\|_{0, \Omega}\right\}\left\|\nabla \boldsymbol{w}_{h}\right\|_{0, \Omega} \\
& \leq C \lambda h L\{1+\lambda h L\}\left\|\left(\boldsymbol{v}_{h}, q_{h}\right)\right\|\left\|\left(\boldsymbol{w}_{h}, t_{h}\right)\right\|,
\end{aligned}
$$

Copyright $@$ by SIAM. Unauthorized reproduction of this article is prohibited. 
and item III is bounded as

$$
\begin{aligned}
\mathrm{III} & =-\sum_{K \in \mathcal{T}_{h}}\left(p_{e}^{K}\left(\lambda\left(\nabla \boldsymbol{v}_{h}\right) \mathcal{I}_{h} \boldsymbol{u}_{\lambda}+\lambda\left(\nabla \mathcal{I}_{h} \boldsymbol{u}_{\lambda}\right) \boldsymbol{v}_{h}\right), p_{e}^{K}\left(\nabla t_{h}\right)\right)_{K} \\
& \leq C \lambda h \sum_{K \in \mathcal{T}_{h}}\left(\left\|\nabla \boldsymbol{v}_{h}\right\|_{0, K}\left\|\mathcal{I}_{h} \boldsymbol{u}_{\lambda}\right\|_{\infty, K}+\left\|\nabla \mathcal{I}_{h} \boldsymbol{u}_{\lambda}\right\|_{0,4, K}\left\|\boldsymbol{v}_{h}\right\|_{0,4, K}\right) h_{K}\left\|\nabla t_{h}\right\|_{0, K} \\
& \leq C \lambda h\left\{\left|\boldsymbol{v}_{h}\right|_{1, \Omega}\left\|\boldsymbol{u}_{\lambda}\right\|_{\infty, \Omega}+\left\|\mathcal{I}_{h} \boldsymbol{u}_{\lambda}\right\|_{1,4, \Omega}\left\|\boldsymbol{v}_{h}\right\|_{0,4, \Omega}\right\}\left\|t_{h}\right\|_{0, \Omega} \\
& \leq C \lambda h L\left\|\left(\boldsymbol{v}_{h}, q_{h}\right)\right\|\left\|\left(\boldsymbol{w}_{h}, t_{h}\right)\right\| .
\end{aligned}
$$

Therefore, from (3.31), (3.32), and (3.33) it follows that

$$
\left\|A_{1}-D_{\boldsymbol{u}, p} F_{h}\left(\lambda, \mathcal{I}_{h} \boldsymbol{u}_{\lambda}, \mathcal{J}_{h} p_{\lambda}\right)\right\|_{\mathcal{L}\left(\boldsymbol{V}_{h} \times Q_{h}\right)} \leq C \lambda h L(1+\lambda h)^{2}(1+\lambda h L),
$$

and the result follows for some $h_{0} \leq h_{1}$ using analogous arguments.

Along with the previous lemma, the next result states further properties of the mapping $F_{h}$ and its derivative.

Lemma 3.9. Assume the hypotheses of Lemma 3.8 hold. Therefore, there exists a constant $C$, which does not depend on $h$ or $\lambda$, such that

$$
\left\|F_{h}\left(\lambda, \mathcal{I}_{h} \boldsymbol{u}_{\lambda}, \mathcal{J}_{h} p_{\lambda}\right)\right\| \leq C h\left\{\lambda L^{2}(1+\lambda h)^{2}\left(1+h+\lambda h^{2} L\right)+L\right\}
$$

Furthermore, for each $\rho>0$ and for all $\left(\boldsymbol{v}_{h}, q_{h}\right) \in \boldsymbol{V}_{h} \times Q_{h}$ such that $\left(\boldsymbol{v}_{h}, q_{h}\right)$ belongs to the ball centered at $\left(\mathcal{I}_{h} \boldsymbol{u}_{\lambda}, \mathcal{J}_{h} p_{\lambda}\right)$ with radius $\rho$, there exists a constant $C>0$, independent of $h$ and $\lambda$ but depending on $\rho$, such that

$$
\begin{aligned}
& \left\|D_{\boldsymbol{u}, p} F_{h}\left(\lambda, \mathcal{I}_{h} \boldsymbol{u}_{\lambda}, \mathcal{J}_{h} p_{\lambda}\right)-D_{\boldsymbol{u}, p} F_{h}\left(\lambda, \boldsymbol{v}_{h}, q_{h}\right)\right\|_{\mathcal{L}\left(\boldsymbol{V}_{h} \times Q_{h}\right)} \\
& \quad \leq C \lambda\left\{(1+\lambda h)^{2}(1+\lambda+\lambda L)\right\}\left\|\left(\mathcal{I}_{h} \boldsymbol{u}_{\lambda}-\boldsymbol{v}_{h}, \mathcal{J}_{h} p_{\lambda}-q_{h}\right)\right\| .
\end{aligned}
$$

Proof. We first note that $F_{h}\left(\lambda, \boldsymbol{u}_{\lambda}, p_{\lambda}\right)=\mathbf{0}$, and then using the linearity of $T_{h}$ we obtain

$$
\begin{aligned}
& \left\|F_{h}\left(\lambda, \mathcal{I}_{h} \boldsymbol{u}_{\lambda}, \mathcal{J}_{h} p_{\lambda}\right)\right\|=\left\|F_{h}\left(\lambda, \mathcal{I}_{h} \boldsymbol{u}_{\lambda}, \mathcal{J}_{h} p_{\lambda}\right)-F_{h}\left(\lambda, \boldsymbol{u}_{\lambda}, p_{\lambda}\right)\right\| \\
& \quad \leq\left\|\left(\mathcal{I}_{h} \boldsymbol{u}_{\lambda}-\boldsymbol{u}_{\lambda}, \mathcal{J}_{h} p_{\lambda}-p_{\lambda}\right)\right\|+\left\|T_{h}\left(G_{h}\left(\lambda, \boldsymbol{u}_{\lambda}, p_{\lambda}\right)-G_{h}\left(\lambda, \mathcal{I}_{h} \boldsymbol{u}_{\lambda}, \mathcal{J}_{h} p_{\lambda}\right)\right)\right\| \\
& \quad=S_{1}+S_{2}
\end{aligned}
$$

To estimate $S_{1}$ we use (3.11) and (3.16) and easily obtain

$$
S_{1} \leq C h L
$$

Copyright (c) by SIAM. Unauthorized reproduction of this article is prohibited. 
Next, using the continuity of $T_{h}$ (cf. Lemma 3.7) and the dual norm (1.4), it holds that

$$
\begin{aligned}
S_{2}= & \left\|T_{h}\left(G_{h}\left(\lambda, \boldsymbol{u}_{\lambda}, p_{\lambda}\right)-G_{h}\left(\lambda, \mathcal{I}_{h} \boldsymbol{u}_{\lambda}, \mathcal{J}_{h} p_{\lambda}\right)\right)\right\| \\
\leq & C(1+\lambda h)^{2} \sup _{\left\|\left(\boldsymbol{v}_{h}, q_{h}\right)\right\| \leq 1}\left(G_{h}\left(\lambda, \boldsymbol{u}_{\lambda}, p_{\lambda}\right)-G_{h}\left(\lambda, \mathcal{I}_{h} \boldsymbol{u}_{\lambda}, \mathcal{J}_{h} p_{\lambda}\right),\left(\boldsymbol{v}_{h}, q_{h}\right)\right) \\
\leq & C(1+\lambda h)^{2} \sup _{\left\|\left(\boldsymbol{v}_{h}, q_{h}\right)\right\| \leq 1} \\
& \left\{\lambda\left(\left(\nabla \boldsymbol{u}_{\lambda}\right) \boldsymbol{u}_{\lambda}-\left(\nabla \mathcal{I}_{h} \boldsymbol{u}_{\lambda}\right) \mathcal{I}_{h} \boldsymbol{u}_{\lambda}, \boldsymbol{v}_{h}\right)_{\Omega}\right. \\
& -\sum_{K \in \mathcal{T}_{h}}\left(p_{e}^{K}\left(\boldsymbol{f}+\Delta \boldsymbol{u}_{\lambda}-\lambda\left(\nabla \boldsymbol{u}_{\lambda}\right) \boldsymbol{u}_{\lambda}-\nabla p_{\lambda}\right), p_{e}^{K}\left(\lambda\left(\nabla \boldsymbol{v}_{h}\right) \boldsymbol{u}_{\lambda}\right)\right)_{K} \\
& \quad-\sum_{K \in \mathcal{T}_{h}}\left(p_{e}^{K}\left(-\boldsymbol{f}+\lambda\left(\nabla \mathcal{I}_{h} \boldsymbol{u}_{\lambda}\right) \mathcal{I}_{h} \boldsymbol{u}_{\lambda}+\nabla \mathcal{J}_{h} p_{\lambda}\right), p_{e}^{K}\left(\lambda\left(\nabla \boldsymbol{v}_{h}\right) \mathcal{I}_{h} \boldsymbol{u}_{\lambda}\right)\right)_{K} \\
& \left.\quad-\sum_{K \in \mathcal{T}_{h}}\left(p_{e}^{K}\left(\lambda\left(\nabla \mathcal{I}_{h} \boldsymbol{u}_{\lambda}\right) \mathcal{I}_{h} \boldsymbol{u}_{\lambda}-\lambda\left(\nabla \boldsymbol{u}_{\lambda}\right) \boldsymbol{u}_{\lambda}\right), p_{e}^{K}\left(\nabla q_{h}\right)\right)_{K}\right\} \\
\leq & C(1+\lambda h)^{2} \sup _{\left\|\left(\boldsymbol{v}_{h}, q_{h}\right)\right\| \leq 1}\{\mathrm{I}+\mathrm{II}+\mathrm{III}+\mathrm{IV}\} .
\end{aligned}
$$

As for the first term, using (3.29) and the Cauchy-Schwarz and Poincaré inequalities, we have

$$
\begin{aligned}
\mathrm{I} & =\lambda\left(\left(\nabla \mathcal{I}_{h} \boldsymbol{u}_{\lambda}\right) \mathcal{I}_{h} \boldsymbol{u}_{\lambda}-\left(\nabla \boldsymbol{u}_{\lambda}\right) \boldsymbol{u}_{\lambda}, \boldsymbol{v}_{h}\right)_{\Omega} \\
& \leq \lambda\left\|\left(\nabla \mathcal{I}_{h} \boldsymbol{u}_{\lambda}\right) \mathcal{I}_{h} \boldsymbol{u}_{\lambda}-\left(\nabla \boldsymbol{u}_{\lambda}\right) \boldsymbol{u}_{\lambda}\right\|_{0, \Omega}\left\|\boldsymbol{v}_{h}\right\|_{0, \Omega} \\
& \leq C \lambda h L^{2}\left\|\left(\boldsymbol{v}_{h}, q_{h}\right)\right\| .
\end{aligned}
$$

Since $\left(\boldsymbol{u}_{\lambda}, p_{\lambda}\right)$ is the solution of problem (3.1), then II $=0$. We are left with bounding III and IV, for which we use Lemma 3.4, (3.15) and the continuous embedding $H^{2}(\Omega) \hookrightarrow L^{\infty}(\Omega)$, and (3.30) as follows:

$$
\begin{aligned}
\mathrm{III} & =-\sum_{K \in \mathcal{T}_{h}}\left(p_{e}^{K}\left(-\boldsymbol{f}+\lambda\left(\nabla \mathcal{I}_{h} \boldsymbol{u}_{\lambda}\right) \mathcal{I}_{h} \boldsymbol{u}_{\lambda}+\nabla \mathcal{J}_{h} p_{\lambda}\right), p_{e}^{K}\left(\lambda\left(\nabla \boldsymbol{v}_{h}\right) \mathcal{I}_{h} \boldsymbol{u}_{\lambda}\right)\right)_{K} \\
& \leq C \sum_{K \in \mathcal{T}_{h}} \lambda h_{K}^{2}\left\|-\boldsymbol{f}+\lambda\left(\nabla \mathcal{I}_{h} \boldsymbol{u}_{\lambda}\right) \mathcal{I}_{h} \boldsymbol{u}_{\lambda}+\nabla \mathcal{J}_{h} p_{\lambda}\right\|_{0, K}\left\|\left(\nabla \boldsymbol{v}_{h}\right) \mathcal{I}_{h} \boldsymbol{u}_{\lambda}\right\|_{0, K} \\
& \leq C \sum_{K \in \mathcal{T}_{h}} \lambda h_{K}^{2}\left\{(1+\lambda h L)\left\|\boldsymbol{u}_{\lambda}\right\|_{2, K}+\left\|p_{\lambda}\right\|_{1, \omega_{K}}\right\}\left|\boldsymbol{v}_{h}\right|_{1, K} L \\
& \leq C \lambda h^{2} L^{2}\{1+\lambda h L\}\left\|\left(\boldsymbol{v}_{h}, q_{h}\right)\right\| .
\end{aligned}
$$

As for item IV, we further use (3.29) and (3.7) to get

$$
\begin{aligned}
\mathrm{IV} & =\sum_{K \in \mathcal{T}_{h}}\left(p_{e}^{K}\left(\lambda\left(\nabla \mathcal{I}_{h} \boldsymbol{u}_{\lambda}\right) \mathcal{I}_{h} \boldsymbol{u}_{\lambda}-\lambda\left(\nabla \boldsymbol{u}_{\lambda}\right) \boldsymbol{u}_{\lambda}\right), p_{e}^{K}\left(\nabla q_{h}\right)\right)_{K} \\
& \leq C \sum_{K \in \mathcal{T}_{h}} \lambda h_{K}\left\|\left(\nabla \mathcal{I}_{h} \boldsymbol{u}_{\lambda}\right) \mathcal{I}_{h} \boldsymbol{u}_{\lambda}-\left(\nabla \boldsymbol{u}_{\lambda}\right) \boldsymbol{u}_{\lambda}\right\|_{0, K}\left\|p_{e}^{K}\left(\nabla q_{h}\right)\right\|_{0, K} \\
& \leq C \sum_{K \in \mathcal{T}_{h}} \lambda L h_{K}^{2}\left\|\boldsymbol{u}_{\lambda}\right\|_{2, K} h_{K}\left\|\nabla q_{h}\right\|_{0, K} \\
& \leq C \lambda h^{2} L^{2}\left\|\left(\boldsymbol{v}_{h}, q_{h}\right)\right\| .
\end{aligned}
$$

Copyright (c) by SIAM. Unauthorized reproduction of this article is prohibited. 
Gathering (3.39)-(3.41), we get

$$
S_{2} \leq C \lambda h L^{2}(1+\lambda h)^{2}\left(1+h+\lambda h^{2} L\right)
$$

and thus, using (3.37) and the bounds given in (3.38) and (3.42), we establish (3.35).

Estimate (3.36) is addressed next. Let $\left(\boldsymbol{u}_{h}, p_{h}\right),\left(\boldsymbol{v}_{h}, q_{h}\right),\left(\boldsymbol{w}_{h}, r_{h}\right) \in \boldsymbol{V}_{h} \times Q_{h}$ with $\left\|\left(\boldsymbol{w}_{h}, r_{h}\right)\right\|=1$. From the stability of the discrete Stokes operator in Lemma 3.7 we get

$$
\begin{aligned}
& \left\|D_{\boldsymbol{u}, p} F_{h}\left(\lambda, \boldsymbol{v}_{h}, q_{h}\right)\left(\boldsymbol{w}_{h}, r_{h}\right)-D_{\boldsymbol{u}, p} F_{h}\left(\lambda, \mathcal{I}_{h} \boldsymbol{u}_{\lambda}, \mathcal{J}_{h} p_{\lambda}\right)\left(\boldsymbol{w}_{h}, r_{h}\right)\right\| \\
& =\left\|T_{h}\left(D_{\boldsymbol{u}, p} G_{h}\left(\lambda, \boldsymbol{v}_{h}, q_{h}\right)\left(\boldsymbol{w}_{h}, r_{h}\right)-D_{\boldsymbol{u}, p} G_{h}\left(\lambda, \mathcal{I}_{h} \boldsymbol{u}_{\lambda}, \mathcal{J}_{h} p_{\lambda}\right)\left(\boldsymbol{w}_{h}, r_{h}\right)\right)\right\| \\
& \leq C(1+\lambda h)^{2} \sup _{\left\|\left(\boldsymbol{z}_{h}, s_{h}\right)\right\| \leq 1}\left(D_{\boldsymbol{u}, p} G_{h}\left(\lambda, \boldsymbol{v}_{h}, q_{h}\right)\left(\boldsymbol{w}_{h}, r_{h}\right)\right. \\
& \left.-D_{\boldsymbol{u}, p} G_{h}\left(\lambda, \mathcal{I}_{h} \boldsymbol{u}_{\lambda}, \mathcal{J}_{h} p_{\lambda}\right)\left(\boldsymbol{w}_{h}, r_{h}\right),\left(\boldsymbol{z}_{h}, s_{h}\right)\right) \\
& \leq C(1+\lambda h)^{2} \sup _{\left\|\left(\boldsymbol{z}_{h}, s_{h}\right)\right\| \leq 1} \\
& \left\{-\left(\lambda\left(\nabla \mathcal{I}_{h} \boldsymbol{u}_{\lambda}\right) \boldsymbol{w}_{h}+\lambda\left(\nabla \boldsymbol{w}_{h}\right) \mathcal{I}_{h} \boldsymbol{u}_{\lambda}, \boldsymbol{z}_{h}\right)+\left(\lambda\left(\nabla \boldsymbol{v}_{h}\right) \boldsymbol{w}_{h}+\lambda\left(\nabla \boldsymbol{w}_{h}\right) \boldsymbol{v}_{h}, \boldsymbol{z}_{h}\right)\right. \\
& +\sum_{K \in \mathcal{T}_{h}}\left(p_{e}^{K}\left(\boldsymbol{f}-\lambda\left(\nabla \mathcal{I}_{h} \boldsymbol{u}_{\lambda}\right) \mathcal{I}_{h} \boldsymbol{u}_{\lambda}-\nabla \mathcal{J}_{h} p_{\lambda}\right), p_{e}^{K}\left(\lambda\left(\nabla \boldsymbol{z}_{h}\right) \boldsymbol{w}_{h}\right)\right)_{K} \\
& -\sum_{K \in \mathcal{T}_{h}}\left(p_{e}^{K}\left(\boldsymbol{f}-\lambda\left(\nabla \boldsymbol{v}_{h}\right) \boldsymbol{v}_{h}-\nabla q_{h}\right), p_{e}^{K}\left(\lambda\left(\nabla \boldsymbol{z}_{h}\right) \boldsymbol{w}_{h}\right)\right)_{K} \\
& -\sum_{K \in \mathcal{T}_{h}}\left(p_{e}^{K}\left(\lambda\left(\nabla \mathcal{I}_{h} \boldsymbol{u}_{\lambda}\right) \boldsymbol{w}_{h}+\lambda\left(\nabla \boldsymbol{w}_{h}\right) \mathcal{I}_{h} \boldsymbol{u}_{\lambda}+\nabla r_{h}\right), p_{e}^{K}\left(\lambda\left(\nabla \boldsymbol{z}_{h}\right) \mathcal{I}_{h} \boldsymbol{u}_{\lambda}\right)\right)_{K} \\
& +\sum_{K \in \mathcal{T}_{h}}\left(p_{e}^{K}\left(\lambda\left(\nabla \boldsymbol{v}_{h}\right) \boldsymbol{w}_{h}+\lambda\left(\nabla \boldsymbol{w}_{h}\right) \boldsymbol{v}_{h}+\nabla r_{h}\right), p_{e}^{K}\left(\lambda\left(\nabla \boldsymbol{z}_{h}\right) \boldsymbol{v}_{h}\right)\right)_{K} \\
& -\sum_{K \in \mathcal{T}_{h}}\left(p_{e}^{K}\left(\lambda\left(\nabla \mathcal{I}_{h} \boldsymbol{u}_{\lambda}\right) \boldsymbol{w}_{h}+\lambda\left(\nabla \boldsymbol{w}_{h}\right) \mathcal{I}_{h} \boldsymbol{u}_{\lambda}\right), p_{e}^{K}\left(\nabla s_{h}\right)\right)_{K} \\
& \left.+\sum_{K \in \mathcal{T}_{h}}\left(p_{e}^{K}\left(\lambda\left(\nabla \boldsymbol{v}_{h}\right) \boldsymbol{w}_{h}+\lambda\left(\nabla \boldsymbol{w}_{h}\right) \boldsymbol{v}_{h}\right), p_{e}^{K}\left(\nabla s_{h}\right)\right)_{K}\right\} \\
& =C(1+\lambda h)^{2} \sup _{\left\|\left(\boldsymbol{z}_{h}, s_{h}\right)\right\| \leq 1}\{\mathrm{~V}+\mathrm{VI}+\mathrm{VII}+\mathrm{VIII}+\mathrm{IX}+\mathrm{X}\} .
\end{aligned}
$$

We bound item $\mathrm{V}$ by using (1.5) as follows:

$$
\begin{aligned}
\mathrm{V} & =-\lambda\left(\left(\nabla\left(\mathcal{I}_{h} \boldsymbol{u}_{\lambda}-\boldsymbol{v}_{h}\right)\right) \boldsymbol{w}_{h}+\left(\nabla \boldsymbol{w}_{h}\right)\left(\mathcal{I}_{h} \boldsymbol{u}_{\lambda}-\boldsymbol{v}_{h}\right), \boldsymbol{z}_{h}\right) \\
& \leq 2 \alpha \lambda\left|\mathcal{I}_{h} \boldsymbol{u}_{\lambda}-\boldsymbol{v}_{h}\right|_{1, \Omega}\left|\boldsymbol{w}_{h}\right|_{1, \Omega}\left|\boldsymbol{z}_{h}\right|_{1, \Omega} \\
& \leq 2 \alpha \lambda\left\|\left(\mathcal{I}_{h} \boldsymbol{u}_{\lambda}-\boldsymbol{v}_{h}, \mathcal{J}_{h} p_{\lambda}-q_{h}\right)\right\|\left\|\left(\boldsymbol{w}_{h}, r_{h}\right)\right\|\left\|\left(\boldsymbol{z}_{h}, s_{h}\right)\right\|,
\end{aligned}
$$

and for item VI we use the Cauchy-Schwarz inequality, Lemma 3.4, (3.5), and (3.7)

Copyright $@$ by SIAM. Unauthorized reproduction of this article is prohibited. 
to obtain

$$
\begin{aligned}
\mathrm{VI} & =-\sum_{K \in \mathcal{T}_{h}}\left(p_{e}^{K}\left(\nabla\left(\mathcal{J}_{h} p_{\lambda}-q_{h}\right)\right), p_{e}^{K}\left(\lambda\left(\nabla \boldsymbol{z}_{h}\right) \boldsymbol{w}_{h}\right)\right)_{K} \\
& \leq C \lambda \sum_{K \in \mathcal{T}_{h}} h_{K}\left\|\nabla\left(\mathcal{J}_{h} p_{\lambda}-q_{h}\right)\right\|_{0, K} h_{K}\left\|\nabla \boldsymbol{z}_{h}\right\|_{0, K}\left\|\boldsymbol{w}_{h}\right\|_{\infty, K} \\
& \leq C \lambda \sum_{K \in \mathcal{T}_{h}}\left\|\mathcal{J}_{h} p_{\lambda}-q_{h}\right\|_{0, K}\left|\boldsymbol{z}_{h}\right|_{1, K}\left\|\boldsymbol{w}_{h}\right\|_{0, K} \\
& \leq C \lambda\left\|\left(\mathcal{I}_{h} \boldsymbol{u}_{\lambda}-\boldsymbol{v}_{h}, \mathcal{J}_{h} p_{\lambda}-q_{h}\right)\right\|\left\|\left(\boldsymbol{z}_{h}, s_{h}\right)\right\|\left\|\left(\boldsymbol{w}_{h}, r_{h}\right)\right\| .
\end{aligned}
$$

Following analogous steps, and using Poincaré's inequality, we can establish the following estimates for items VII-X:

$$
\begin{aligned}
& \mathrm{VII}=-\sum_{K \in \mathcal{T}_{h}} \lambda\left(p_{e}^{K}\left(\left(\nabla \mathcal{I}_{h} \boldsymbol{u}_{\lambda}\right) \mathcal{I}_{h} \boldsymbol{u}_{\lambda}-\left(\nabla \boldsymbol{v}_{h}\right) \boldsymbol{v}_{h}\right), p_{e}^{K}\left(\lambda\left(\nabla \boldsymbol{z}_{h}\right) \boldsymbol{w}_{h}\right)\right)_{K} \\
& \leq C \lambda^{2} \sum_{K \in \mathcal{T}_{h}} h_{K}^{2}\left\|\left(\nabla \mathcal{I}_{h} \boldsymbol{u}_{\lambda}\right) \mathcal{I}_{h} \boldsymbol{u}_{\lambda}-\left(\nabla \boldsymbol{v}_{h}\right) \boldsymbol{v}_{h}\right\|_{0, K}\left\|\left(\nabla \boldsymbol{z}_{h}\right) \boldsymbol{w}_{h}\right\|_{0, K} \\
& \leq C \lambda^{2} \sum_{K \in \mathcal{T}_{h}} h_{K}\left\|\left(\nabla \mathcal{I}_{h} \boldsymbol{u}_{\lambda}\right) \mathcal{I}_{h} \boldsymbol{u}_{\lambda}-\left(\nabla \boldsymbol{v}_{h}\right) \boldsymbol{v}_{h}\right\|_{0, K}\left|\boldsymbol{z}_{h}\right|_{1, K}\left\|\boldsymbol{w}_{h}\right\|_{0, K} \\
& \leq C \lambda^{2}\left\{\sum_{K \in \mathcal{T}_{h}} h_{K}\left\|\left(\nabla \mathcal{I}_{h} \boldsymbol{u}_{\lambda}\right)\left(\mathcal{I}_{h} \boldsymbol{u}_{\lambda}-\boldsymbol{v}_{h}\right)\right\|_{0, K}\right. \\
& \left.+\sum_{K \in \mathcal{T}_{h}} h_{K}\left\|\left(\nabla\left(\mathcal{I}_{h} \boldsymbol{u}_{\lambda}-\boldsymbol{v}_{h}\right)\right) \boldsymbol{v}_{h}\right\|_{0, K}\right\}\left|\boldsymbol{z}_{h}\right|_{1, \Omega}\left|\boldsymbol{w}_{h}\right|_{1, \Omega} \\
& \leq C \lambda^{2}\left\{\sum_{K \in \mathcal{T}_{h}} h_{K}\left\|\nabla \mathcal{I}_{h} \boldsymbol{u}_{\lambda}\right\|_{0, K}\left\|\mathcal{I}_{h} \boldsymbol{u}_{\lambda}-\boldsymbol{v}_{h}\right\|_{\infty, K}\right. \\
& \left.+\sum_{K \in \mathcal{T}_{h}} h_{K}\left\|\nabla\left(\mathcal{I}_{h} \boldsymbol{u}_{\lambda}-\boldsymbol{v}_{h}\right)\right\|_{0, K}\left\|\boldsymbol{v}_{h}\right\|_{\infty, K}\right\}\left|\boldsymbol{z}_{h}\right|_{1, \Omega}\left|\boldsymbol{w}_{h}\right|_{1, \Omega} \\
& \leq C \lambda^{2}\left\{\left(\sum_{K \in \mathcal{T}_{h}}\left\|\nabla \mathcal{I}_{h} \boldsymbol{u}_{\lambda}\right\|_{0, K}^{2}\right)^{1 / 2}\left(\sum_{K \in \mathcal{T}_{h}}\left\|\mathcal{I}_{h} \boldsymbol{u}_{\lambda}-\boldsymbol{v}_{h}\right\|_{0, K}^{2}\right)^{1 / 2}\right. \\
& \left.+\left(\sum_{K \in \mathcal{T}_{h}}\left|\mathcal{I}_{h} \boldsymbol{u}_{\lambda}-\boldsymbol{v}_{h}\right|_{1, K}^{2}\right)^{1 / 2}\left(\sum_{K \in \mathcal{T}_{h}}\left\|\boldsymbol{v}_{h}\right\|_{0, K}^{2}\right)^{1 / 2}\right\}\left|\boldsymbol{z}_{h}\right|_{1, \Omega}\left|\boldsymbol{w}_{h}\right|_{1, \Omega} \\
& \leq C \lambda^{2}\left\{\left\|\boldsymbol{u}_{\lambda}\right\|_{2, \Omega}+\left|\boldsymbol{v}_{h}\right|_{1, \Omega}\right\}\left\|\left(\mathcal{I}_{h} \boldsymbol{u}_{\lambda}-\boldsymbol{v}_{h}, \mathcal{J}_{h} p_{\lambda}-q_{h}\right)\right\|\|\|\left(\boldsymbol{w}_{h}, r_{h}\right)\|\|\left(\boldsymbol{z}_{h}, s_{h}\right) \| \\
& (3.45) \leq C \lambda^{2}\{L+\rho\}\left\|\left(\mathcal{I}_{h} \boldsymbol{u}_{\lambda}-\boldsymbol{v}_{h}, \mathcal{J}_{h} p_{\lambda}-q_{h}\right)\right\|\left\|\left(\boldsymbol{w}_{h}, r_{h}\right)\right\|\left\|\left(\boldsymbol{z}_{h}, s_{h}\right)\right\|,
\end{aligned}
$$

Copyright (c) by SIAM. Unauthorized reproduction of this article is prohibited. 
and

$$
\begin{aligned}
& \mathrm{VIII}=-\sum_{K \in \mathcal{T}_{h}} \lambda\left(p_{e}^{K}\left(\left(\nabla\left(\mathcal{I}_{h} \boldsymbol{u}_{\lambda}-\boldsymbol{v}_{h}\right)\right) \boldsymbol{w}_{h}+\left(\nabla \boldsymbol{w}_{h}\right)\left(\mathcal{I}_{h} \boldsymbol{u}_{\lambda}-\boldsymbol{v}_{h}\right)\right), p_{e}^{K}\left(\lambda\left(\nabla \boldsymbol{z}_{h}\right) \mathcal{I}_{h} \boldsymbol{u}_{\lambda}\right)\right)_{K} \\
& \leq C \lambda^{2} \sum_{K \in \mathcal{T}_{h}} h_{K}^{2}\left\|\left(\nabla\left(\mathcal{I}_{h} \boldsymbol{u}_{\lambda}-\boldsymbol{v}_{h}\right)\right) \boldsymbol{w}_{h}+\left(\nabla \boldsymbol{w}_{h}\right)\left(\mathcal{I}_{h} \boldsymbol{u}_{\lambda}-\boldsymbol{v}_{h}\right)\right\|_{0, K}\left\|\left(\nabla \boldsymbol{z}_{h}\right) \mathcal{I}_{h} \boldsymbol{u}_{\lambda}\right\|_{0, K} \\
& \leq C \lambda^{2} \sum_{K \in \mathcal{T}_{h}}\left\{\left|\mathcal{I}_{h} \boldsymbol{u}_{\lambda}-\boldsymbol{v}_{h}\right|_{1, K}\left\|\boldsymbol{w}_{h}\right\|_{0, K}+\left|\boldsymbol{w}_{h}\right|_{1, K} \| \mathcal{I}_{h} \boldsymbol{u}_{\lambda}\right. \\
&\left.\quad-\boldsymbol{v}_{h} \|_{0, K}\right\}\left|\boldsymbol{z}_{h}\right|_{1, K}\left\|\mathcal{I}_{h} \boldsymbol{u}_{\lambda}\right\|_{0, K} \\
& \leq C \lambda^{2} L\left\|\left(\mathcal{I}_{h} \boldsymbol{u}_{\lambda}-\boldsymbol{v}_{h}, \mathcal{J}_{h} p_{\lambda}-q_{h}\right)\right\|\left\|\left(\boldsymbol{w}_{h}, r_{h}\right)\right\|\left\|\left(\boldsymbol{z}_{h}, s_{h}\right)\right\|,
\end{aligned}
$$

and

$$
\begin{aligned}
& \mathrm{IX}=-\sum_{K \in \mathcal{T}_{h}} \lambda\left(p_{e}^{K}\left(\left(\nabla \boldsymbol{v}_{h}\right) \boldsymbol{w}_{h}+\left(\nabla \boldsymbol{w}_{h}\right) \boldsymbol{v}_{h}+\nabla r_{h}\right), p_{e}^{K}\left(\lambda\left(\nabla \boldsymbol{z}_{h}\right)\left(\mathcal{I}_{h} \boldsymbol{u}_{\lambda}-\boldsymbol{v}_{h}\right)\right)\right)_{K} \\
& \leq C \lambda^{2} \sum_{K \in \mathcal{T}_{h}} h_{K}^{2}\left\|\left(\nabla \boldsymbol{v}_{h}\right) \boldsymbol{w}_{h}+\left(\nabla \boldsymbol{w}_{h}\right) \boldsymbol{v}_{h}+\nabla r_{h}\right\|_{0, K}\left\|\left(\nabla \boldsymbol{z}_{h}\right)\left(\mathcal{I}_{h} \boldsymbol{u}_{\lambda}-\boldsymbol{v}_{h}\right)\right\|_{0, K} \\
& \leq C \lambda^{2} \sum_{K \in \mathcal{T}_{h}}\left\{\left|\boldsymbol{v}_{h}\right|_{1, K}\left\|\boldsymbol{w}_{h}\right\|_{0, K}+\left|\boldsymbol{w}_{h}\right|_{1, K}\left\|\boldsymbol{v}_{h}\right\|_{0, K}\right. \\
&\left.\quad+\left\|r_{h}\right\|_{0, K}\right\}\left|\boldsymbol{z}_{h}\right|_{1, K}\left\|\mathcal{I}_{h} \boldsymbol{u}_{\lambda}-\boldsymbol{v}_{h}\right\|_{0, K} \\
& \leq C \lambda^{2}\left|\boldsymbol{v}_{h}\right|_{1, \Omega}\left\|\left(\mathcal{I}_{h} \boldsymbol{u}_{\lambda}-\boldsymbol{v}_{h}, \mathcal{J}_{h} p_{\lambda}-q_{h}\right)\right\|\left\|\left(\boldsymbol{w}_{h}, r_{h}\right)\right\|\left\|\left(\boldsymbol{z}_{h}, s_{h}\right)\right\| \\
& \leq C \lambda^{2}\{L+\rho\}\left\|\left(\mathcal{I}_{h} \boldsymbol{u}_{\lambda}-\boldsymbol{v}_{h}, \mathcal{J}_{h} p_{\lambda}-q_{h}\right)\right\|\left\|\left(\boldsymbol{w}_{h}, r_{h}\right)\right\|\left\|\left(\boldsymbol{z}_{h}, s_{h}\right)\right\|,
\end{aligned}
$$

and

$$
\begin{aligned}
\mathrm{X} & =-\sum_{K \in \mathcal{T}_{h}} \lambda\left(p_{e}^{K}\left(\left(\nabla\left(\mathcal{I}_{h} \boldsymbol{u}_{\lambda}-\boldsymbol{v}_{h}\right)\right) \boldsymbol{w}_{h}+\left(\nabla \boldsymbol{w}_{h}\right)\left(\mathcal{I}_{h} \boldsymbol{u}_{\lambda}-\boldsymbol{v}_{h}\right)\right), p_{e}^{K}\left(\nabla s_{h}\right)\right)_{K} \\
& \leq C \lambda \sum_{K \in \mathcal{T}_{h}} h_{K}\left\|\left(\nabla\left(\mathcal{I}_{h} \boldsymbol{u}_{\lambda}-\boldsymbol{v}_{h}\right)\right) \boldsymbol{w}_{h}+\left(\nabla \boldsymbol{w}_{h}\right)\left(\mathcal{I}_{h} \boldsymbol{u}_{\lambda}-\boldsymbol{v}_{h}\right)\right\|_{0, K} h_{K}\left\|\nabla s_{h}\right\|_{0, K} \\
& \leq C \lambda\left(\left|\mathcal{I}_{h} \boldsymbol{u}_{\lambda}-\boldsymbol{v}_{h}\right|_{1, \Omega}\left\|\boldsymbol{w}_{h}\right\|_{0, \Omega}+\left|\boldsymbol{w}_{h}\right|_{1, \Omega}\left\|\mathcal{I}_{h} \boldsymbol{u}_{\lambda}-\boldsymbol{v}_{h}\right\|_{0, \Omega}\right)\left\|s_{h}\right\|_{0, \Omega} \\
(3.48) & \leq C \lambda\left\|\left(\mathcal{I}_{h} \boldsymbol{u}_{\lambda}-\boldsymbol{v}_{h}, \mathcal{J}_{h} p_{\lambda}-q_{h}\right)\right\|\left\|\left(\boldsymbol{z}_{h}, s_{h}\right)\right\|\left\|\left(\boldsymbol{w}_{h}, r_{h}\right)\right\| .
\end{aligned}
$$

Finally, gathering (3.43)-(3.48) the estimate (3.36) follows.

We are now ready to prove the existence and uniqueness of a local branch of discrete solutions and to present the main error estimate.

Theorem 3.10. Assume the hypotheses of Lemma 3.8 hold. Therefore, there is a positive constant $h_{0}(\Lambda)$ such that for all $h$ with $0<h \leq h_{0}$ a unique branch $\lambda \rightarrow\left(\boldsymbol{u}_{h, \lambda}, p_{h, \lambda}\right)$ of solutions of problem (3.4) exists in a neighborhood of $\left(\boldsymbol{u}_{\lambda}, p_{\lambda}\right)$. Moreover, the following estimate holds:

$$
\sup _{\lambda \in \Lambda}\left\{\left|\boldsymbol{u}_{\lambda}-\boldsymbol{u}_{h, \lambda}\right|_{1, \Omega}^{2}+\left\|p_{\lambda}-p_{h, \lambda}\right\|_{0, \Omega}^{2}\right\}^{1 / 2} \leq C h,
$$

where $C=C(L, \Lambda)>0$ does not depend on $h$.

Proof. From Lemma 3.8 we have that $D_{\boldsymbol{u}, p} F_{h}\left(\lambda, \mathcal{I}_{h} \boldsymbol{u}_{\lambda}, \mathcal{J}_{h} p_{\lambda}\right)$ is an isomorphism of $\boldsymbol{V}_{h} \times Q_{h}$ onto itself for each $\lambda \in \Lambda$, provided that $h \sup _{\lambda \in \Lambda} \lambda$ is sufficiently small.

Copyright $@$ by SIAM. Unauthorized reproduction of this article is prohibited. 
In addition, from (3.36) (cf. Lemma 3.9) we obtain that

$$
\begin{array}{r}
\left\|F_{h}\left(\lambda, \boldsymbol{v}_{h}, q_{h}\right)-F_{h}\left(\lambda, \boldsymbol{w}_{h}, t_{h}\right)-D_{\boldsymbol{u}, p} F_{h}\left(\lambda, \mathcal{I}_{h} \boldsymbol{u}_{\lambda}, \mathcal{J}_{h} p_{\lambda}\right)\left[\left(\boldsymbol{v}_{h}, q_{h}\right)-\left(\boldsymbol{w}_{h}, t_{h}\right)\right]\right\| \\
\leq C(\lambda, L) \rho\left\|\left(\boldsymbol{v}_{h}, q_{h}\right)-\left(\boldsymbol{w}_{h}, t_{h}\right)\right\|
\end{array}
$$

for all $\left(\boldsymbol{v}_{h}, q_{h}\right),\left(\boldsymbol{w}_{h}, t_{h}\right) \in B\left(\left(\mathcal{I}_{h} \boldsymbol{u}_{\lambda}, \mathcal{J}_{h} p_{\lambda}\right) ; \rho\right)$. The two above facts constitute the hypothesis of Theorem IV.3.6 in [17]. Hence, supposing that $h$ is small enough such as

$$
4 C(\lambda, L)\left\|\left\{D_{\boldsymbol{u}, p} F_{h}\left(\lambda, \mathcal{I}_{h} \boldsymbol{u}_{\lambda}, \mathcal{J}_{h} p_{\lambda}\right)\right\}^{-1}\right\|_{\mathcal{L}\left(\boldsymbol{V}_{h} \times Q_{h}\right)}^{2}\left\|F_{h}\left(\lambda, \mathcal{I}_{h} \boldsymbol{u}_{\lambda}, \mathcal{J}_{h} p_{\lambda}\right)\right\|<1
$$

and applying (3.35) (cf. Lemma 3.9) and Theorem IV.3.6 in [17] we conclude that problem (3.28) has a unique solution $\left(\boldsymbol{u}_{h, \lambda}, p_{h, \lambda}\right) \in B\left(\left(\mathcal{I}_{h} \boldsymbol{u}_{\lambda}, \mathcal{J}_{h} p_{\lambda}\right) ; \rho\right)$, where $\rho$ is given by

$$
\rho:=2\left\|\left\{D_{\boldsymbol{u}, p} F_{h}\left(\lambda, \mathcal{I}_{h} \boldsymbol{u}_{\lambda}, \mathcal{J}_{h} p_{\lambda}\right)\right\}^{-1}\right\|_{\mathcal{L}\left(\boldsymbol{V}_{h} \times Q_{h}\right)}\left\|F_{h}\left(\lambda, \mathcal{I}_{h} \boldsymbol{u}_{\lambda}, \mathcal{J}_{h} p_{\lambda}\right)\right\| \leq C(L, \Lambda) h,
$$

and the result follows using (3.11), (3.16), and the triangular inequality.

Remark 3. It is worth noticing at this point that the same results hold for the simplified RELP method (2.4), since the operator $p_{e}^{K} \circ \Pi_{K}$ satisfies the exact same properties as $p_{e}^{K}$. In particular, Lemma 3.4 is valid for the former, and then all the above proofs can be easily adapted to treat the simplified method.

We finish this section by briefly addressing the issue of how to recover a divergencefree velocity field. We suppose that the finite element space for the pressure consists of discontinuous functions and modify the approach from $[1,2]$ slightly by defining in each $K \in \mathcal{T}_{h}$

$$
\boldsymbol{u}_{n c, \lambda}=\sum_{F \subset \partial K \cap \Omega} \tilde{\tau}_{F} \Pi_{F}\left(\llbracket \partial_{\boldsymbol{n}} \boldsymbol{u}_{h, \lambda}+p_{h, \lambda} \boldsymbol{n} \rrbracket\right) \cdot \boldsymbol{n} \varphi_{F},
$$

where $\varphi_{F}:= \pm \frac{h_{F}}{2|K|}\left(\boldsymbol{x}-\boldsymbol{x}_{F}\right)$, with $\boldsymbol{x}_{F}$ the opposite node to the edge $F$, stands for the lowest order Raviart-Thomas basis function. Using (3.49), we build the nonconforming velocity field $\hat{\boldsymbol{u}}_{h, \lambda}:=\boldsymbol{u}_{h, \lambda}+\boldsymbol{u}_{n c, \lambda}$ which is pointwise divergence-free and shares the same convergence properties as $\boldsymbol{u}_{h, \lambda}$. This is stated in the following result.

Lemma 3.11. Let us suppose that the discrete space for the pressure consists of discontinuous functions. If $\boldsymbol{u}_{h, \lambda}$ is the solution of (3.4) and $\boldsymbol{u}_{n c, \lambda}$ is given by (3.49), then the velocity field $\hat{\boldsymbol{u}}_{h, \lambda}:=\boldsymbol{u}_{h, \lambda}+\boldsymbol{u}_{n c, \lambda}$ satisfies

$$
\nabla \cdot \hat{\boldsymbol{u}}_{h, \lambda}=0 \quad \text { in each } K \in \mathcal{T}_{h} .
$$

Moreover, under the hypothesis of Theorem 3.10, there exists a constant $C=C(L, \Lambda)>$ 0 , independent of $h$, such that

$$
\sup _{\lambda \in \Lambda}\left\{\sum_{K \in \mathcal{T}_{h}}\left|\boldsymbol{u}_{\lambda}-\hat{\boldsymbol{u}}_{h, \lambda}\right|_{1, K}^{2}\right\}^{1 / 2} \leq C h .
$$

Proof. The proof of the first part is a slight variation of Lemma 3.8 in [1], and hence we omit it. The error estimate reduces to prove a bound on $\left|\boldsymbol{u}_{n c, \lambda}\right|_{1, K}$ which, after using the fact that $\tilde{\tau}_{F} \leq C h_{F}$ (see [2]), follows the same steps as in [1], Lemma 3.9. 


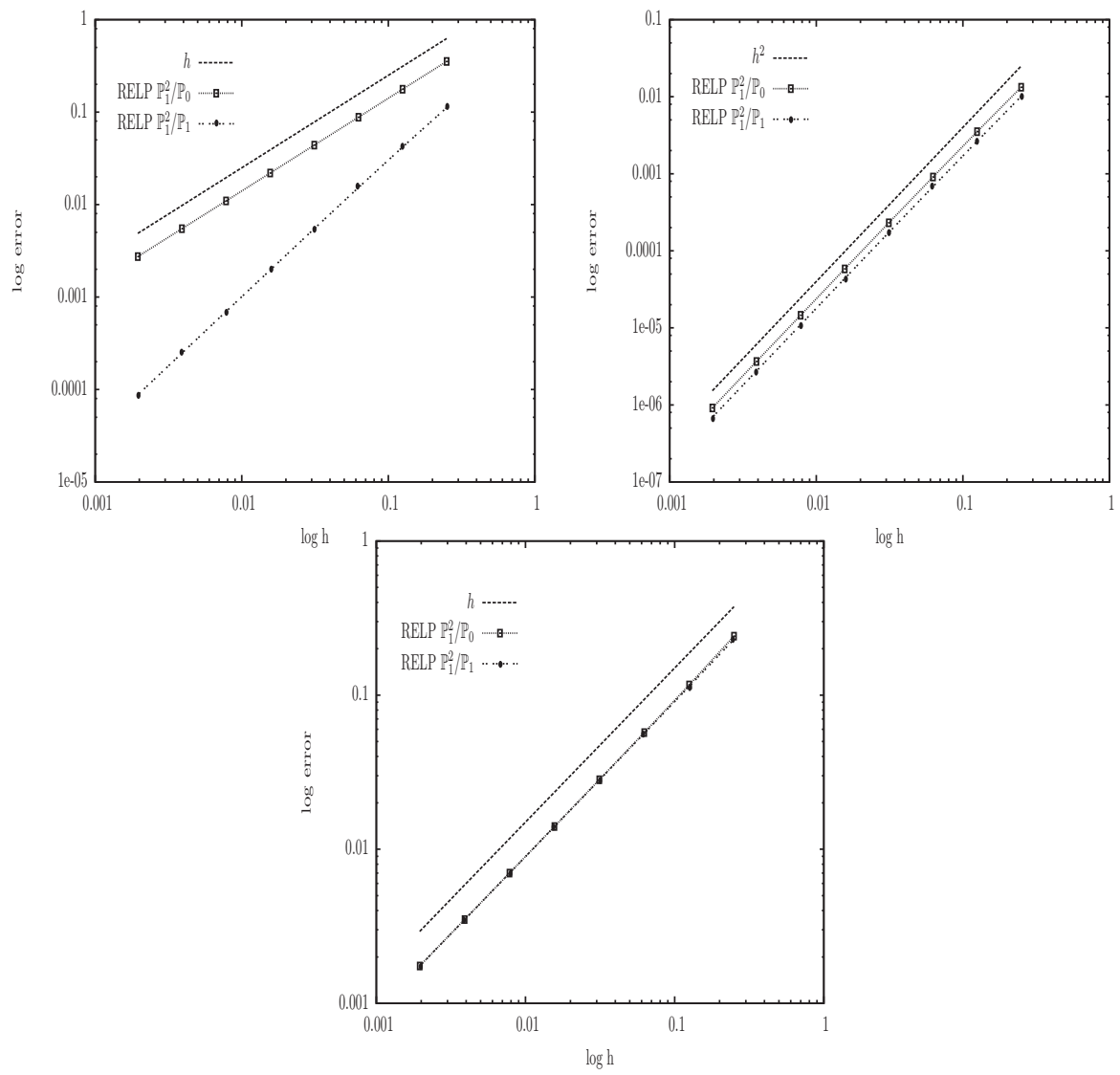

FIG. 4.1. Convergence history of $\left\|p-p_{h}\right\|_{0, \Omega}$ (top left), $\left\|\boldsymbol{u}-\boldsymbol{u}_{h}\right\|_{0, \Omega}$ (top right), and $\left|\boldsymbol{u}-\boldsymbol{u}_{h}\right|_{1, \Omega}$ (bottom). Here $\nu=1$.

4. Numerical validations. This section is devoted to testing the new RELP method (2.4) having as discrete spaces the pairs $\mathbb{P}_{1}^{2} / \mathbb{P}_{0}$ and $\mathbb{P}_{1}^{2} / \mathbb{P}_{1}$ (with continuous pressures). In what follows, we first validate theoretical convergence rates through a benchmark with an analytical solution. Next, we address more involved problems representing a fluid flow around a circular cylinder and the standard lid-driven cavity problem in the high Reynolds number setting, which constitute a more challenging assessment of the present method.

4.1. A study of convergence. The domain is set to be the unit square $\Omega=$ $(0,1) \times(0,1)$. We choose $\nu=1$ and $\nu=10^{-2}$, and $\boldsymbol{f}$ is such as the exact solution $\boldsymbol{u}(x, y):=\left(u_{1}(x, y), u_{2}(x, y)\right)$ and $p(x, y)$ of the Navier-Stokes equations is given by

$$
u_{1}(x, y):=e^{x} \sin (y), \quad u_{2}(x, y):=e^{x} \cos (y), \quad p(x, y):=-\frac{1}{2} e^{2 x}+\frac{1}{4}\left(e^{2}-1\right) .
$$

We remark that the velocity is a harmonic function and then the RELP method is fully consistent for the element $\mathbb{P}_{1}^{2} / \mathbb{P}_{1}$. In Figures 4.1-4.2 we provide the convergence history using the norms $\left\|p-p_{h}\right\|_{0, \Omega},\left\|\boldsymbol{u}-\boldsymbol{u}_{h}\right\|_{0, \Omega}$, and $\left|\boldsymbol{u}-\boldsymbol{u}_{h}\right|_{1, \Omega}$, where $\left(\boldsymbol{u}_{h}, p_{h}\right)$ is the solution of (2.4).

We observe that the numerical orders show a perfect agreement with the theoretical ones, plus a second order convergence for the pressure for the $\mathbb{P}_{1}^{2} / \mathbb{P}_{1}$ case. 

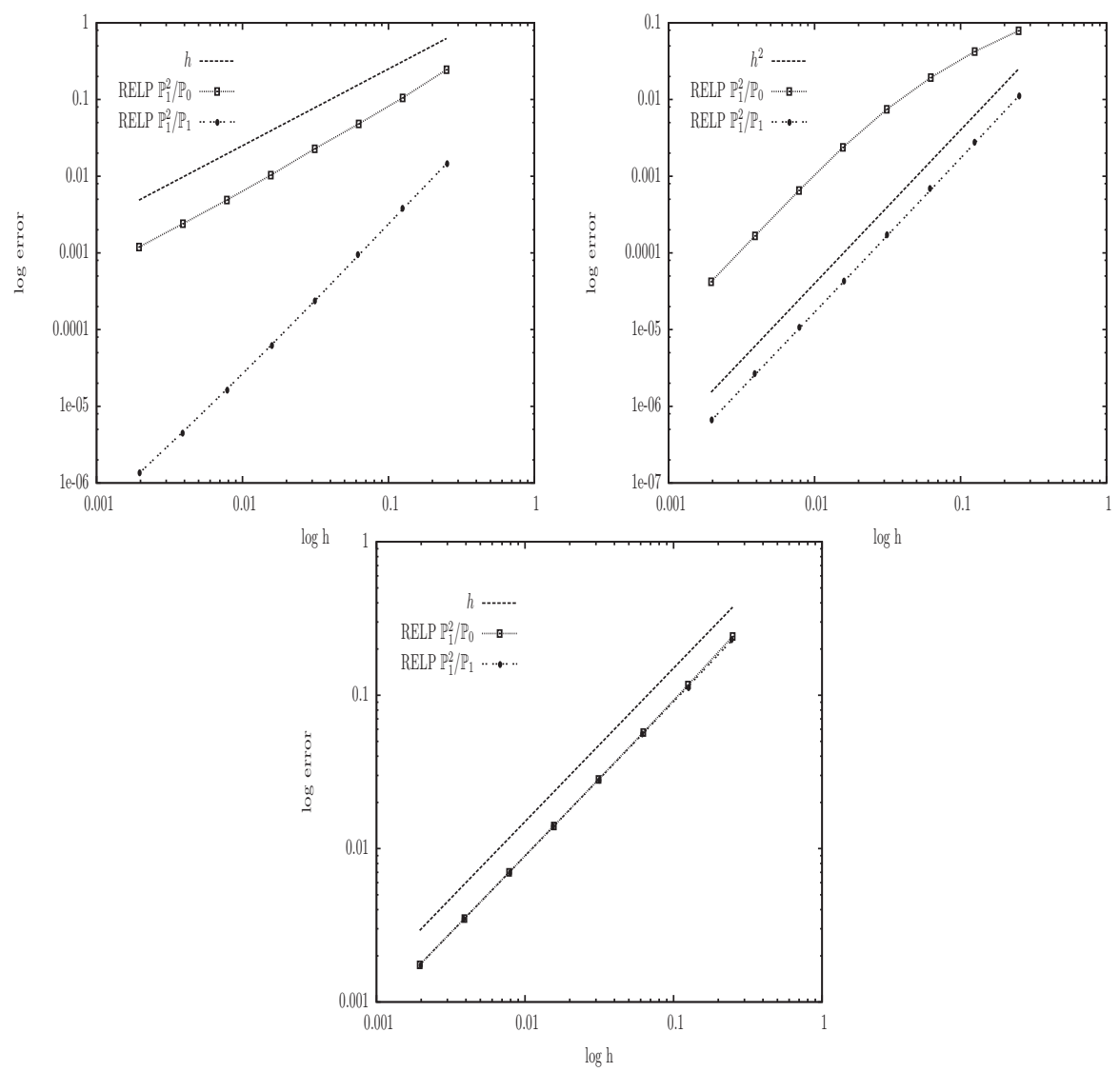

FIG. 4.2. Convergence history of $\left\|p-p_{h}\right\|_{0, \Omega}$ (top left), $\left\|\boldsymbol{u}-\boldsymbol{u}_{h}\right\|_{0, \Omega}$ (top right), and $\left|\boldsymbol{u}-\boldsymbol{u}_{h}\right|_{1, \Omega}$ (bottom). Here $\nu=10^{-2}$.

TABLE 4.1

Error in the divergence, $\max _{K \in \mathcal{T}_{h}}\left|\nabla \cdot \hat{\boldsymbol{u}}_{h}\right|_{K} \mid$, for $\mathbb{P}_{1} / \mathbb{P}_{0}$.

\begin{tabular}{|c||c|c|c|c|c|}
\hline$h$ & 0.25 & 0.125 & 0.0625 & 0.03125 & 0.015625 \\
\hline$\nu=1$ & $8 \times 10^{-15}$ & $4.9 \times 10^{-14}$ & $2.4 \times 10^{-13}$ & $1.4 \times 10^{-12}$ & $5.7 \times 10^{-12}$ \\
\hline$\nu=10^{-2}$ & $9.1 \times 10^{-12}$ & $2.6 \times 10^{-11}$ & $2.4 \times 10^{-11}$ & $8 \times 10^{-11}$ & $2.6 \times 10^{-13}$ \\
\hline
\end{tabular}

Next, for the element $\mathbb{P}_{1}^{2} / \mathbb{P}_{0}$ we update the solution $\boldsymbol{u}_{h}$ with (3.49) and produce the divergence-free velocity field $\hat{\boldsymbol{u}}_{h}=\boldsymbol{u}_{h}+\boldsymbol{u}_{n c}$. The results are given in Table 4.1 assuming different values for $\nu$. The procedure preserves the optimality of the error as pointed out in Figure 4.3, in agreement with the theory.

4.2. The circular cylinder problem. The setting of the problem is given in $[21,25]$ with the inflow velocity field set as $\left(1.2 y(0.41-y) / 0.41^{2}, 0\right)^{T}$ and the viscosity $\nu=10^{-3}$. We assess the accuracy of the RELP method through its capacity to correctly approximate some quantities of interest such as the drag $\left(C_{D}\right)$, the difference of pressure $(\Delta p)$ measured at two points on the cylinder, and the maximum length of the vortices behind the cylinder $\left(L_{r}\right)$. These quantities are compared to the ones obtained from others numerical schemes in Table 4.2, where the results show that the 

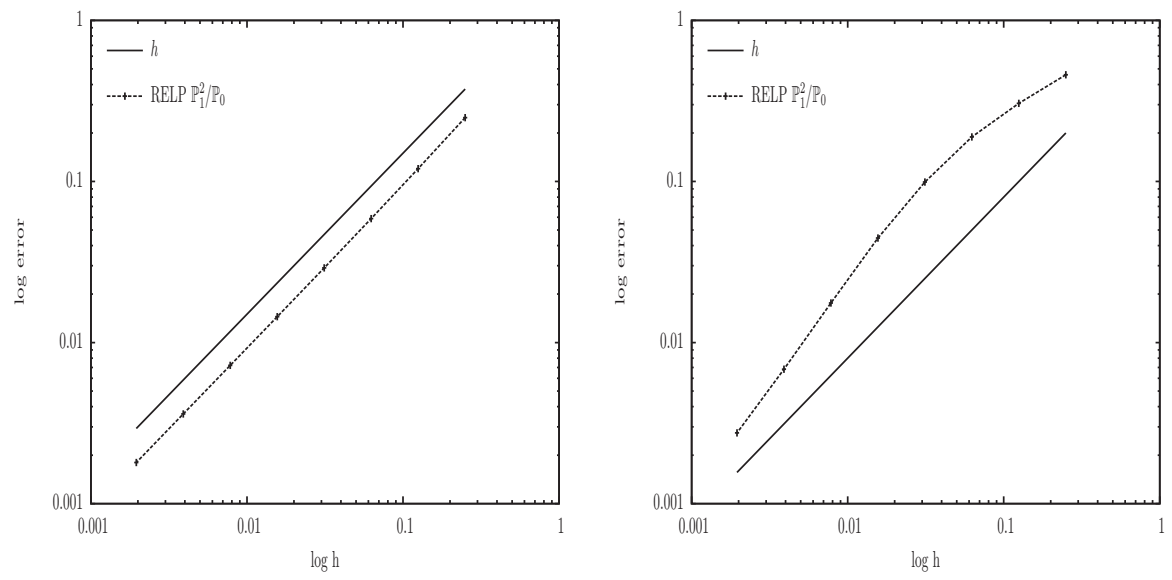

FIG. 4.3. Convergence history of $\left[\sum_{K \in \mathcal{T}_{h}}\left|\boldsymbol{u}-\boldsymbol{u}_{h}-\boldsymbol{u}_{n c}\right|_{1, K}^{2}\right]^{1 / 2}$ for the updated velocity $\hat{\boldsymbol{u}}_{h}$ with the element $\mathbb{P}_{1}^{2} / \mathbb{P}_{0}$. Here $\nu=1$ (left) and $\nu=10^{-2}$ (right).

TABLE 4.2

Assessment of the RELP method.

\begin{tabular}{||l||l|l|l|}
\hline & $C_{D}$ & $\Delta p$ & $L_{r}$ \\
\hline \hline Schäfer and Turek [21] & 5.58 & 0.1175 & 0.085 \\
\hline Medic and Mohammadi [19] & 5.65 & 0.121 & 0.082 \\
\hline RELP $\mathbb{P}_{1}^{2} \times \mathbb{P}_{1}$ & 5.54 & 0.1171 & 0.083 \\
\hline RELP $\mathbb{P}_{1}^{2} \times \mathbb{P}_{0}$ & 5.46 & 0.1149 & 0.084 \\
\hline
\end{tabular}
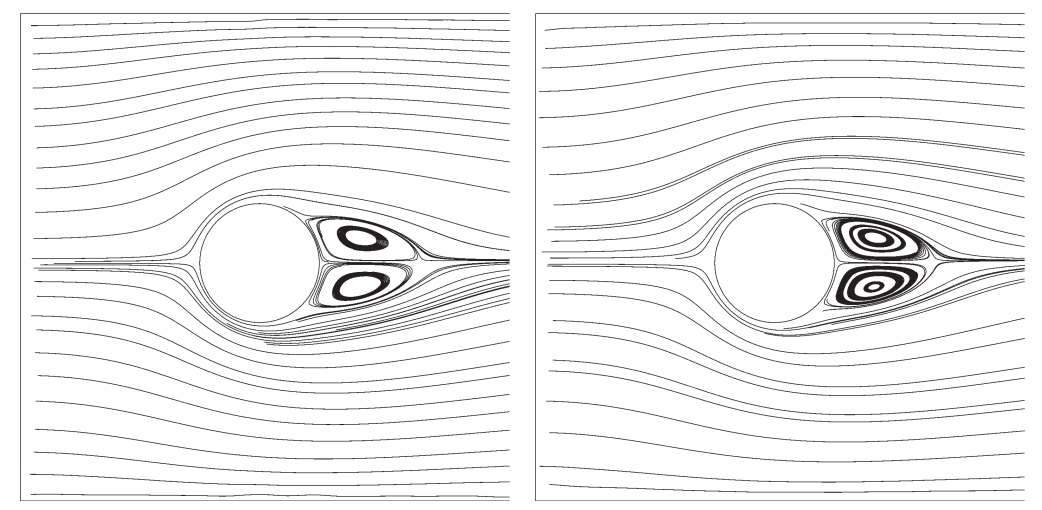

FIG. 4.4. Streamlines around the cylinder with $\mathbb{P}_{1}^{2} \times \mathbb{P}_{0}$ (left) and $\mathbb{P}_{1}^{2} \times \mathbb{P}_{1}$ (right).

present method provides very competitive results. Figure 4.4 depicts the streamlines of the flow around the cylinder using the $\mathbb{P}_{1}^{2} \times \mathbb{P}_{0}$ and $\mathbb{P}_{1}^{2} \times \mathbb{P}_{1}$ elements.

4.3. The lid-driven cavity flow. Next, a challenging test involving a high Reynolds number flow is addressed. More precisely, we attempt to solve the liddriven cavity problem with $\boldsymbol{f}=\mathbf{0}$ and $\nu=\frac{1}{5000}$ and consider a structured mesh of 


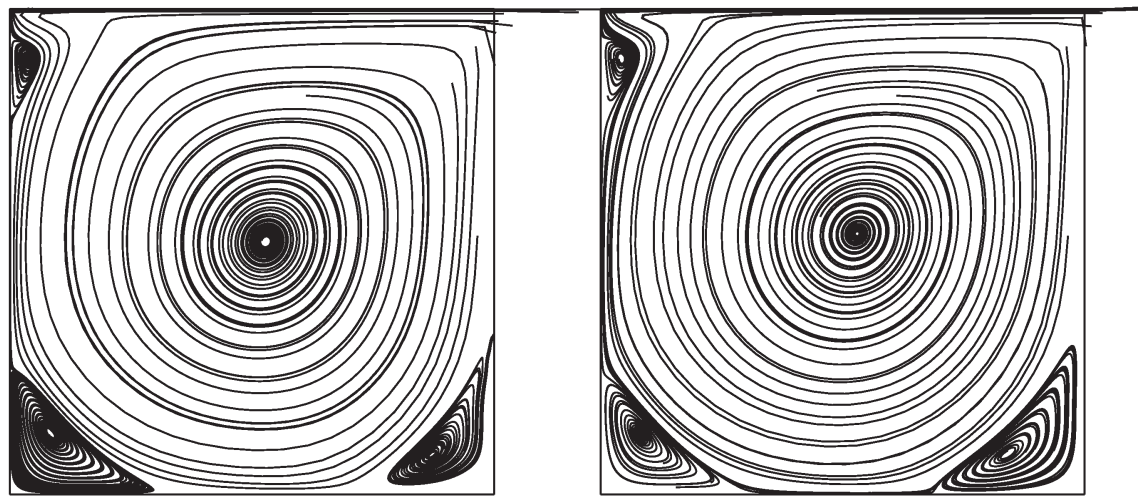

FIG. 4.5. Streamlines of the solution for $\mathbb{P}_{1}^{2} \times \mathbb{P}_{0}$ (left) and $\mathbb{P}_{1}^{2} \times \mathbb{P}_{1}$ (right) schemes. The Reynolds number is 5000.

TABLE 4.3

Primary vortex center position.

\begin{tabular}{||c||c|c|c|c||}
\hline$R e$ & Ghia, Ghia, and Shin [16] & NSIKE [19] & RELP $\mathbb{P}_{1}^{2} / \mathbb{P}_{0}$ & RELP $\mathbb{P}_{1}^{2} / \mathbb{P}_{1}$ \\
\hline 5000 & $x=0.5117$ & $x=0.53$ & $x=0.5285$ & $x=0.5298$ \\
& $y=0.5352$ & $y=0.53$ & $y=0.521$ & $y=0.5370$ \\
\hline
\end{tabular}

around 65,000 elements. We depict in Figure 4.5 the streamlines obtained using both pairs of interpolation spaces. We can see that the method provides a well-balanced dose of numerical diffusion as the secondary vortices are recovered. Also, the precision of the RELP method is validated comparing the numerical solutions provided by the present method with previously available reference solutions. We can see in Table 4.3 that such results are in accordance even if this example lies outside the theory.

5. Conclusion. New RELP methods made stable the simplest and lowest equal order pairs of interpolation spaces for the fully nonlinear Navier-Stokes equations. In the process of proving well-posedness and optimal convergence in the natural norms, a new stabilized method for the Stokes model was also introduced and analyzed. In addition, a simplified version of the RELP method was presented. This simplified method shares the same desired properties of the original method, avoids the use of two level approaches, and is computationally competitive. Next, this method was combined with a simple postprocessing procedure to produce a locally conservative solution which is optimally convergent in the discontinuous pressure case. As such, the methods in this work may be seen as an appealing alternative to simulate complex flows using the cheapest and simplest elements in a precise way while respecting the divergence-free constraint exactly.

Appendix A. The discrete Stokes operator. The discrete Stokes operator includes a formally new stabilized finite element method, given by the following: Find $\left(\boldsymbol{u}_{h}, p_{h}\right) \in \boldsymbol{V}_{h} \times Q_{h}$ such that

$$
\boldsymbol{B}\left(\left(\boldsymbol{u}_{h}, p_{h}\right),\left(\boldsymbol{v}_{h}, q_{h}\right)\right)=\left\langle\boldsymbol{w}, \boldsymbol{v}_{h}\right\rangle+\left(r, q_{h}\right)
$$


for all $\left(\boldsymbol{v}_{h}, q_{h}\right) \in \boldsymbol{V}_{h} \times Q_{h}$, where $(\boldsymbol{w}, r) \in \boldsymbol{V}^{\prime} \times Q$ is given, and $\boldsymbol{B}(.,$.$) reads as follows:$

$$
\begin{aligned}
\boldsymbol{B}\left(\left(\boldsymbol{u}_{h}, p_{h}\right),\left(\boldsymbol{v}_{h}, q_{h}\right)\right):= & \left(\nabla \boldsymbol{u}_{h}, \nabla \boldsymbol{v}_{h}\right)-\left(p_{h}, \nabla \cdot \boldsymbol{v}_{h}\right)+\left(q_{h}, \nabla \cdot \boldsymbol{u}_{h}\right) \\
& +\sum_{K \in \mathcal{T}_{h}}\left(p_{e}^{K}\left(\nabla p_{h}\right), p_{e}^{K}\left(\nabla q_{h}\right)\right)_{K} \\
& +\left(\lambda \chi_{h}\left(\boldsymbol{x} \nabla \cdot \boldsymbol{u}_{h}\right), \lambda \chi_{h}\left(\boldsymbol{x} \nabla \cdot \boldsymbol{v}_{h}\right)\right)_{K} \\
& +\sum_{F \in \mathcal{E}_{h}} \frac{h_{F}}{12}\left(\llbracket \partial_{\boldsymbol{n}} \boldsymbol{u}_{h}+p_{h} \boldsymbol{n} \rrbracket, \llbracket \partial_{\boldsymbol{n}} \boldsymbol{v}_{h}+q_{h} \boldsymbol{n} \rrbracket\right)_{F} .
\end{aligned}
$$

The next result establishes the existence and unicity of solution for (A.1).

Lemma A.1. The mapping $T_{h}$ is well defined.

Proof. Defining the mesh-dependent norm

$$
\begin{aligned}
\left\|\left(\boldsymbol{v}_{h}, q_{h}\right)\right\|_{h}^{2}:= & \left|\boldsymbol{v}_{h}\right|_{1, \Omega}^{2}+\sum_{K \in \mathcal{T}_{h}}\left\|\chi_{h}\left(q_{h}\right)\right\|_{0, K}^{2}+\left\|\lambda \chi_{h}\left(\boldsymbol{x} \nabla \cdot \boldsymbol{v}_{h}\right)\right\|_{0, K}^{2} \\
& +\sum_{F \in \mathcal{E}_{h}} \frac{h_{F}}{12}\left\|\llbracket \partial_{\boldsymbol{n}} \boldsymbol{v}_{h}+q_{h} \boldsymbol{n} \rrbracket\right\|_{0, F}^{2},
\end{aligned}
$$

and using (2.3) it is easy to realize that for all $\left(\boldsymbol{v}_{h}, q_{h}\right) \in \boldsymbol{V}_{h} \times Q_{h}$

$$
\boldsymbol{B}\left(\left(\boldsymbol{v}_{h}, q_{h}\right),\left(\boldsymbol{v}_{h}, q_{h}\right)\right)=\left\|\left(\boldsymbol{v}_{h}, q_{h}\right)\right\|_{h}^{2},
$$

and thus the problem (A.1) is well-posed and the operator $T_{h}$ is well defined.

Lemma A.2. The operator $T_{h}$ is continuous. More precisely, there exists $C>0$, independent of $h$ and $\lambda$ such that

$$
\left\|T_{h}(\boldsymbol{w}, r)\right\| \leq C(1+\lambda h)^{2}\|(\boldsymbol{w}, r)\|_{\left(\boldsymbol{V}_{h} \times Q_{h}\right)^{\prime}}
$$

for all $(\boldsymbol{w}, r) \in(\boldsymbol{V} \times Q)^{\prime}$.

Proof. The proof follows standard arguments, but we present it here for completeness. Let $\left(\boldsymbol{u}_{h}, p_{h}\right)=T_{h}(\boldsymbol{w}, r)$. From (A.3) we see that

$$
\begin{aligned}
\left\|\left(\boldsymbol{u}_{h}, p_{h}\right)\right\|_{h}^{2} & =\boldsymbol{B}\left(\left(\boldsymbol{u}_{h}, p_{h}\right),\left(\boldsymbol{u}_{h}, p_{h}\right)\right)=\left\langle\boldsymbol{w}, \boldsymbol{u}_{h}\right\rangle+\left(r, p_{h}\right) \\
& \leq\|\boldsymbol{w}\|_{\boldsymbol{V}_{h}^{\prime}}\left|\boldsymbol{u}_{h}\right|_{1, \Omega}+\|r\|_{Q_{h}^{\prime}}\left\|p_{h}\right\|_{0, \Omega} .
\end{aligned}
$$

To bound the $L^{2}(\Omega)$-norm of $p_{h}$, let $\boldsymbol{z} \in H_{0}^{1}(\Omega)^{2}$ be such that

$$
\beta\left\|p_{h}\right\|_{0, \Omega}|\boldsymbol{z}|_{1, \Omega} \leq\left(p_{h}, \nabla \cdot \boldsymbol{z}\right)
$$

Copyright $@$ by SIAM. Unauthorized reproduction of this article is prohibited. 
and let $\boldsymbol{z}_{h}$ be the Clément interpolate of $\boldsymbol{z}$. Then, integrating by parts, using that $\left(\boldsymbol{u}_{h}, p_{h}\right)$ is the solution of (A.1), (3.16), and (3.17) we arrive at

$$
\begin{aligned}
\beta\left\|p_{h}\right\|_{0, \Omega}|\boldsymbol{z}|_{1, \Omega} \leq & \left(p_{h}, \nabla \cdot\left(\boldsymbol{z}-\boldsymbol{z}_{h}\right)\right)+\left(p_{h}, \nabla \cdot \boldsymbol{z}_{h}\right) \\
= & -\sum_{K \in \mathcal{T}_{h}}\left(\nabla p_{h}, \boldsymbol{z}-\boldsymbol{z}_{h}\right)_{K}+\sum_{F \in \mathcal{E}_{h}}\left(\llbracket p_{h} \boldsymbol{n} \rrbracket, \boldsymbol{z}-\boldsymbol{z}_{h}\right)_{F}+\left(\nabla \boldsymbol{u}_{h}, \nabla \boldsymbol{z}_{h}\right) \\
& +\sum_{K \in \mathcal{T}_{h}}\left(\lambda \chi_{h}\left(\boldsymbol{x} \nabla \cdot \boldsymbol{u}_{h}\right), \lambda \chi_{h}\left(\boldsymbol{x} \nabla \cdot \boldsymbol{z}_{h}\right)\right)_{K} \\
& +\sum_{F \in \mathcal{E}_{h}} \frac{h_{F}}{12}\left(\llbracket \partial_{\boldsymbol{n}} \boldsymbol{u}_{h}+p_{h} \boldsymbol{n} \rrbracket, \llbracket \partial_{\boldsymbol{n}} \boldsymbol{z}_{h} \rrbracket\right)_{F}-\left\langle\boldsymbol{w}, \boldsymbol{z}_{h}\right\rangle \\
\leq & C \sum_{K \in \mathcal{T}_{h}} h_{K}\left\|\nabla p_{h}\right\|_{0, K}|\boldsymbol{z}|_{1, \omega_{K}}+C \sum_{F \in \mathcal{E}_{h}} h_{F}^{1 / 2}\left\|\llbracket p_{h} \boldsymbol{n} \rrbracket\right\|_{0, F}|\boldsymbol{z}|_{1, \omega_{F}} \\
& +\left|\boldsymbol{u}_{h}\right|_{1, \Omega}\left|\boldsymbol{z}_{h}\right|_{1, \Omega}+\|\boldsymbol{w}\|_{\boldsymbol{V}_{h}^{\prime}}\left|\boldsymbol{z}_{h}\right|_{1, \Omega} \\
& +C \sum_{K \in \mathcal{T}_{h}} \lambda\left\|\chi_{h}\left(\boldsymbol{x} \nabla \cdot \boldsymbol{u}_{h}\right)\right\|_{0, K} \lambda\left\|\chi_{h}\left(\boldsymbol{x} \nabla \cdot \boldsymbol{z}_{h}\right)\right\|_{0, K} \\
& +\sum_{F \in \mathcal{E}_{h}} \frac{h_{F}}{12}\left\|\llbracket \partial_{\boldsymbol{n}} \boldsymbol{u}_{h}+p_{h} \boldsymbol{n} \rrbracket\right\|_{0, F}\left\|\llbracket \partial_{\boldsymbol{n}} \boldsymbol{z}_{h} \rrbracket\right\|_{0, F} .
\end{aligned}
$$

Next, using the generalized Poincaré's inequality and the fact that $|\boldsymbol{x}|_{1, K} \leq C h_{K}$ and (3.16) we obtain

$$
\left\|\chi_{h}\left(\boldsymbol{x} \nabla \cdot \boldsymbol{z}_{h}\right)\right\|_{0, K}=\frac{\left\|\chi_{h}(\boldsymbol{x})\right\|_{0, K}}{|K|^{1 / 2}}\left\|\nabla \cdot \boldsymbol{z}_{h}\right\|_{0, K} \leq C h_{K}\|\boldsymbol{z}\|_{1, \omega_{K}},
$$

and then (3.6), (3.7), (A.6), and the mesh regularity lead to

$$
\begin{aligned}
\beta\left\|p_{h}\right\|_{0, \Omega}|\boldsymbol{z}|_{1, \Omega} \leq & C\left\{\sum_{K \in \mathcal{T}_{h}} h_{K}^{2}\left\|\nabla p_{h}\right\|_{0, K}^{2}+\sum_{F \in \mathcal{E}_{h}} h_{F}\left\|\llbracket p_{h} \boldsymbol{n} \rrbracket\right\|_{0, F}^{2}\right. \\
& \left.+\left\|\left(\boldsymbol{u}_{h}, p_{h}\right)\right\|_{h}^{2}+\|\boldsymbol{w}\|_{\boldsymbol{V}_{h}^{\prime}}^{2}\right\}^{1 / 2} \\
& \left\{|\boldsymbol{z}|_{1, \Omega}^{2}+\sum_{K \in \mathcal{T}_{h}} \lambda^{2} h_{K}^{2}\|\boldsymbol{z}\|_{1, \omega_{K}}^{2}\right\}^{1 / 2} \\
\leq & C\left\{\left\|\left(\boldsymbol{u}_{h}, p_{h}\right)\right\|_{h}^{2}+\|\boldsymbol{w}\|_{\boldsymbol{V}_{h}^{\prime}}^{2}\right\}^{1 / 2}(1+\lambda h)|\boldsymbol{z}|_{1, \Omega},
\end{aligned}
$$

and dividing by $|\boldsymbol{z}|_{1, \Omega}$ we arrive at

$$
\left\|p_{h}\right\|_{0, \Omega} \leq C(1+\lambda h)\left\{\left\|\left(\boldsymbol{u}_{h}, p_{h}\right)\right\|_{h}^{2}+\|\boldsymbol{w}\|_{\boldsymbol{V}_{h}^{\prime}}^{2}\right\}^{1 / 2} .
$$

Then, using (A.9) in (A.4), and $a b \leq a^{2}+\frac{1}{4} b^{2}$ with $a, b \in \mathbb{R}^{+}$, we arrive at

$$
\begin{aligned}
\left\|\left(\boldsymbol{u}_{h}, p_{h}\right)\right\|_{h}^{2} & \leq C\left(\|\boldsymbol{w}\|_{\boldsymbol{V}_{h}^{\prime}}^{2}+(1+\lambda h)^{2}\|r\|_{Q_{h}^{\prime}}^{2}\right) \\
& \leq C(1+\lambda h)^{2}\left(\|\boldsymbol{w}\|_{\boldsymbol{V}_{h}^{\prime}}^{2}+\|r\|_{Q_{h}^{\prime}}^{2},\right.
\end{aligned}
$$

Copyright $@$ by SIAM. Unauthorized reproduction of this article is prohibited. 
and then replacing this in (A.9) it holds that

$$
\left\|p_{h}\right\|_{0, \Omega} \leq C(1+\lambda h)^{2}\left(\|\boldsymbol{w}\|_{\boldsymbol{V}_{h}^{\prime}}+\|r\|_{Q_{h}^{\prime}}\right)
$$

Finally, the proof ends remarking that $\left\|\left(\boldsymbol{u}_{h}, p_{h}\right)\right\| \leq\left(\left\|\left(\boldsymbol{u}_{h}, p_{h}\right)\right\|_{h}^{2}+\left\|p_{h}\right\|_{0, \Omega}^{2}\right)^{1 / 2}$ and using $\|\boldsymbol{w}\|_{\boldsymbol{V}_{h}^{\prime}}+\|r\|_{Q_{h}^{\prime}} \leq C\|(\boldsymbol{w}, r)\|_{\left(\boldsymbol{V}_{h} \times Q_{h}\right)^{\prime}}$.

LEMmA A.3. There exists a constant $C>0$, independent of $h$ and $\lambda$ such that for all $\boldsymbol{w} \in L^{2}(\Omega)^{2}$ it holds that

$$
\left\|\left(T-T_{h}\right)(\boldsymbol{w}, 0)\right\| \leq C(1+\lambda h)^{2} h\|\boldsymbol{w}\|_{0, \Omega} .
$$

Proof. Let $(\boldsymbol{u}, p)=T(\boldsymbol{w}, 0)$ and $\left(\boldsymbol{u}_{h}, p_{h}\right)=T_{h}(\boldsymbol{w}, 0)$. The proof reduces to prove the error estimates

$$
\begin{aligned}
\left\|\left(\boldsymbol{u}-\boldsymbol{u}_{h}, p-p_{h}\right)\right\|_{h} & \leq C(1+\lambda h) h\left(\|\boldsymbol{u}\|_{2, \Omega}+\|p\|_{1, \Omega}\right), \\
\left\|p-p_{h}\right\|_{0, \Omega} & \leq C(1+\lambda h)^{2} h\left(\|\boldsymbol{u}\|_{2, \Omega}+\|p\|_{1, \Omega}\right)
\end{aligned}
$$

and then applying classical regularity results for the Stokes problem (cf. [17]). To prove (A.12) we split the error into interpolation error $\left(\eta^{\boldsymbol{u}}, \eta^{p}\right):=\left(\boldsymbol{u}-\mathcal{I}_{h}(\boldsymbol{u}), p-\mathcal{J}_{h}(p)\right)$ and discrete error $\left(e_{h}^{\boldsymbol{u}}, e_{h}^{p}\right):=\left(\mathcal{I}_{h}(\boldsymbol{u})-\boldsymbol{u}_{h}, \mathcal{J}_{h}(p)-p_{h}\right)$. Then, using the stability and approximation properties of $\chi_{h}$, the fact that $\boldsymbol{u}$ is solenoidal, (3.11)-(3.17), and the mesh regularity we obtain

$$
\begin{aligned}
\left\|\left(\eta^{\boldsymbol{u}}, \eta^{p}\right)\right\|_{h}^{2}= & \left|\eta^{\boldsymbol{u}}\right|_{1, \Omega}^{2}+\sum_{K \in \mathcal{T}_{h}}\left\|\chi_{h}\left(\eta^{p}\right)\right\|_{0, K}^{2}+\left\|\lambda \chi_{h}\left(\boldsymbol{x} \nabla \cdot \eta^{\boldsymbol{u}}\right)\right\|_{0, K}^{2} \\
& +\sum_{F \in \mathcal{E}_{h}} \frac{h_{F}}{12}\left\|\llbracket \partial_{\boldsymbol{n}} \eta^{\boldsymbol{u}}+\eta^{p} \boldsymbol{n} \rrbracket\right\|_{0, F}^{2} \\
\leq & C h^{2}|\boldsymbol{u}|_{2, \Omega}^{2}+\left\|\eta^{p}\right\|_{0, \Omega}^{2}+\sum_{K \in \mathcal{T}_{h}} \frac{\lambda^{2}\left\|\chi_{h}(\boldsymbol{x})\right\|_{0, K}^{2}}{|K|}\left\|\nabla \cdot \mathcal{I}_{h}(\boldsymbol{u})\right\|_{0, K}^{2} \\
& +C \sum_{F \in \mathcal{E}_{h}} h_{F}^{2}\left(|\boldsymbol{u}|_{2, \omega_{F}}^{2}+|p|_{1, \omega_{F}}^{2}\right) \\
\leq & C h^{2}\left(|\boldsymbol{u}|_{2, \Omega}^{2}+|p|_{1, \Omega}^{2}\right)+C \sum_{K \in \mathcal{T}_{h}} \lambda^{2} h_{K}^{2}\left\|\nabla \cdot \eta^{\boldsymbol{u}}\right\|_{0, K}^{2} \\
\leq & C h^{2}\left(\left(1+\lambda^{2} h^{2}\right)|\boldsymbol{u}|_{2, \Omega}^{2}+|p|_{1, \Omega}^{2}\right) .
\end{aligned}
$$

Next, using arguments very close to the analysis from [1] we can prove that

$$
\left\|\left(e_{h}^{\boldsymbol{u}}, e_{h}^{p}\right)\right\|_{h} \leq C(1+\lambda h) h\left(|\boldsymbol{u}|_{2, \Omega}+|p|_{1, \Omega}\right),
$$

and then (A.12) follows using the triangle inequality. To prove (A.13) we use the continuous inf-sup condition and the definition of the method. We omit further details and refer to [1] for an analysis that can be easily adapted to the present case.

Appendix B. Uniqueness of the discrete solution. We start assuming that $\lambda$ is small enough so that (3.4) reduces to (3.27). Let $\left(\boldsymbol{u}_{h, \lambda}, p_{h, \lambda}\right) \in \boldsymbol{V}_{h} \times Q_{h}$ be a solution of (3.27), and observe that from (3.28), $\left(\boldsymbol{u}_{h, \lambda}, p_{h, \lambda}\right)$ corresponds to a fixed point of the operator $-T_{h} G_{h}$. The proof then reduces to prove that the operator $-T_{h} G_{h}$ is a strict contraction in $B:=\left\{\left(\boldsymbol{v}_{h}, q_{h}\right) \in \boldsymbol{V}_{h} \times Q_{h}:\left\|\left(\boldsymbol{v}_{h}, q_{h}\right)\right\| \leq 1\right\}$ and then use Banach's fixed point theorem.

Copyright (c) by SIAM. Unauthorized reproduction of this article is prohibited. 
Let $\left(\boldsymbol{u}_{h}, p_{h}\right),\left(\boldsymbol{v}_{h}, q_{h}\right) \in B$. Using Lemma 3.7 and the definition of operators $T_{h}$ and $G_{h}$, it holds that

$$
\begin{aligned}
& \left\|T_{h} G_{h}\left(\lambda, \boldsymbol{u}_{h}, p_{h}\right)-T_{h} G_{h}\left(\lambda, \boldsymbol{v}_{h}, q_{h}\right)\right\| \\
& =\left\|T_{h}\left(G_{h}\left(\lambda, \boldsymbol{u}_{h}, p_{h}\right)-G_{h}\left(\lambda, \boldsymbol{v}_{h}, q_{h}\right)\right)\right\| \\
& \leq C(1+\lambda h)^{2} \sup _{\left\|\left(\boldsymbol{w}_{h}, t_{h}\right)\right\| \leq 1}\left(G_{h}\left(\lambda, \boldsymbol{u}_{h}, p_{h}\right)-G_{h}\left(\lambda, \boldsymbol{v}_{h}, q_{h}\right),\left(\boldsymbol{w}_{h}, t_{h}\right)\right) \\
& \leq C(1+\lambda h)^{2} \sup _{\left\|\left(\boldsymbol{w}_{h}, t_{h}\right)\right\| \leq 1} \\
& \left\{\lambda\left(\left(\nabla \boldsymbol{u}_{h}\right) \boldsymbol{u}_{h}-\left(\nabla \boldsymbol{v}_{h}\right) \boldsymbol{v}_{h}, \boldsymbol{w}_{h}\right)\right. \\
& -\sum_{K \in \mathcal{T}_{h}}\left(p_{e}^{K}\left(\boldsymbol{f}-\lambda\left(\nabla \boldsymbol{u}_{h}\right) \boldsymbol{u}_{h}-\nabla p_{h}\right), p_{e}^{K}\left(\lambda\left(\nabla \boldsymbol{w}_{h}\right) \boldsymbol{u}_{h}\right)\right)_{K} \\
& -\sum_{K \in \mathcal{T}_{h}}\left(p_{e}^{K}\left(-\boldsymbol{f}+\lambda\left(\nabla \boldsymbol{v}_{h}\right) \boldsymbol{v}_{h}+\nabla q_{h}\right), p_{e}^{K}\left(\lambda\left(\nabla \boldsymbol{w}_{h}\right) \boldsymbol{v}_{h}\right)\right)_{K} \\
& \left.-\sum_{K \in \mathcal{T}_{h}}\left(p_{e}^{K}\left(\lambda\left(\nabla \boldsymbol{v}_{h}\right) \boldsymbol{v}_{h}-\lambda\left(\nabla \boldsymbol{u}_{h}\right) \boldsymbol{u}_{h}\right), p_{e}^{K}\left(\nabla t_{h}\right)\right)_{K}\right\} \\
& \leq C(1+\lambda h)^{2} \sup _{\left\|\left(\boldsymbol{w}_{h}, t_{h}\right)\right\| \leq 1} \\
& \left\{\lambda\left(\left(\nabla \boldsymbol{u}_{h}\right) \boldsymbol{u}_{h}-\left(\nabla \boldsymbol{v}_{h}\right) \boldsymbol{v}_{h}, \boldsymbol{w}_{h}\right)\right. \\
& -\sum_{K \in \mathcal{T}_{h}}\left(p_{e}^{K}\left(\boldsymbol{f}-\lambda\left(\nabla \boldsymbol{u}_{h}\right) \boldsymbol{u}_{h}-\nabla p_{h}\right), p_{e}^{K}\left(\lambda\left(\nabla \boldsymbol{w}_{h}\right)\left(\boldsymbol{u}_{h}-\boldsymbol{v}_{h}\right)\right)\right)_{K} \\
& -\sum_{K \in \mathcal{T}_{h}}\left(p_{e}^{K}\left(-\lambda\left(\left(\nabla \boldsymbol{u}_{h}\right) \boldsymbol{u}_{h}-\left(\nabla \boldsymbol{v}_{h}\right) \boldsymbol{v}_{h}\right)-\nabla\left(p_{h}-q_{h}\right)\right), p_{e}^{K}\left(\lambda\left(\nabla \boldsymbol{w}_{h}\right) \boldsymbol{v}_{h}\right)\right)_{K} \\
& \left.-\sum_{K \in \mathcal{T}_{h}}\left(p_{e}^{K}\left(\lambda\left(\nabla \boldsymbol{v}_{h}\right) \boldsymbol{v}_{h}-\lambda\left(\nabla \boldsymbol{u}_{h}\right) \boldsymbol{u}_{h}\right), p_{e}^{K}\left(\nabla t_{h}\right)\right)_{K}\right\} \\
& (\text { B.1 })=C(1+\lambda h)^{2} \sup _{\left\|\left(\boldsymbol{w}_{h}, t_{h}\right)\right\| \leq 1}\{\mathrm{I}+\mathrm{II}+\mathrm{III}+\mathrm{IV}\} .
\end{aligned}
$$

Regarding item I above, we use (1.5) and the definition of the norm $\|\cdot\|$ to get

$$
\begin{aligned}
\mathrm{I} & =\lambda\left(\left(\nabla \boldsymbol{u}_{h}\right) \boldsymbol{u}_{h}-\left(\nabla \boldsymbol{v}_{h}\right) \boldsymbol{v}_{h}, \boldsymbol{w}_{h}\right) \\
& =\lambda\left(\left(\nabla\left(\boldsymbol{u}_{h}-\boldsymbol{v}_{h}\right)\right) \boldsymbol{u}_{h}-\left(\nabla \boldsymbol{v}_{h}\right)\left(\boldsymbol{v}_{h}-\boldsymbol{u}_{h}\right), \boldsymbol{w}_{h}\right) \\
& \leq \alpha \lambda\left\{\left|\boldsymbol{u}_{h}-\boldsymbol{v}_{h}\right|_{1, \Omega}\left|\boldsymbol{u}_{h}\right|_{1, \Omega}\left|\boldsymbol{w}_{h}\right|_{1, \Omega}+\left|\boldsymbol{v}_{h}\right|_{1, \Omega}\left|\boldsymbol{u}_{h}-\boldsymbol{v}_{h}\right|_{1, \Omega}\left|\boldsymbol{w}_{h}\right|_{1, \Omega}\right\} \\
& \leq \alpha \lambda\left\|\left(\boldsymbol{u}_{h}, p_{h}\right)-\left(\boldsymbol{v}_{h}, q_{h}\right)\right\|\left\|\left(\boldsymbol{w}_{h}, t_{h}\right)\right\|
\end{aligned}
$$

To bound item II, we employ (3.5), (3.7), (3.19), the Hölder and Cauchy-Schwarz 
inequalities, and the definition of the norm $\|\cdot\|$ as follows:

$$
\begin{aligned}
& \mathrm{II}=-\sum_{K \in \mathcal{T}_{h}}\left(p_{e}^{K}\left(\boldsymbol{f}-\lambda\left(\nabla \boldsymbol{u}_{h}\right) \boldsymbol{u}_{h}-\nabla p_{h}\right), p_{e}^{K}\left(\lambda\left(\nabla \boldsymbol{w}_{h}\right)\left(\boldsymbol{u}_{h}-\boldsymbol{v}_{h}\right)\right)\right)_{K} \\
& \leq C \sum_{K \in \mathcal{T}_{h}} \lambda h_{K}^{2}\left\|\boldsymbol{f}-\lambda\left(\nabla \boldsymbol{u}_{h}\right) \boldsymbol{u}_{h}-\nabla p_{h}\right\|_{0, K}\left\|\left(\nabla \boldsymbol{w}_{h}\right)\left(\boldsymbol{u}_{h}-\boldsymbol{v}_{h}\right)\right\|_{0, K} \\
& \leq C \sum_{K \in \mathcal{T}_{h}} \lambda h_{K}^{2}\left\{\|\boldsymbol{f}\|_{0, K}+\lambda\left\|\nabla \boldsymbol{u}_{h}\right\|_{0, K}\left\|\boldsymbol{u}_{h}\right\|_{\infty, K}\right. \\
&\left.\quad+\left\|\nabla p_{h}\right\|_{0, K}\right\}\left\|\nabla \boldsymbol{w}_{h}\right\|_{\infty, K}\left\|\boldsymbol{u}_{h}-\boldsymbol{v}_{h}\right\|_{0, K} \\
& \leq C \lambda \sum_{K \in \mathcal{T}_{h}}\left\{h_{K}\|\boldsymbol{f}\|_{0, K}+\lambda\left\|\nabla \boldsymbol{u}_{h}\right\|_{0, K}\left\|\boldsymbol{u}_{h}\right\|_{0, K}+\left\|p_{h}\right\|_{0, K}\right\}\left|\boldsymbol{w}_{h}\right|_{1, K}\left\|\boldsymbol{u}_{h}-\boldsymbol{v}_{h}\right\|_{0, K} \\
& \leq C \lambda\left\{h\|\boldsymbol{f}\|_{0, \Omega}+\lambda\left\|\left(\boldsymbol{u}_{h}, p_{h}\right)\right\|^{2}+\left\|\left(\boldsymbol{u}_{h}, p_{h}\right)\right\|\right\}\left\|\left(\boldsymbol{u}_{h}, p_{h}\right)-\left(\boldsymbol{v}_{h}, q_{h}\right)\right\|\left\|\left(\boldsymbol{w}_{h}, t_{h}\right)\right\| \\
&(\mathrm{B} .3) \leq C \lambda\left\{h\|\boldsymbol{f}\|_{0, \Omega}+\lambda+1\right\}\left\|\left(\boldsymbol{u}_{h}, p_{h}\right)-\left(\boldsymbol{v}_{h}, q_{h}\right)\right\|\left\|\left(\boldsymbol{w}_{h}, t_{h}\right)\right\| .
\end{aligned}
$$

The terms III and IV are bounded using arguments similar to the ones used for II, thus giving

$$
\begin{aligned}
\mathrm{III}= & \left.-\sum_{K \in \mathcal{T}_{h}}\left(p_{e}^{K}\left(-\lambda\left(\left(\nabla \boldsymbol{u}_{h}\right) \boldsymbol{u}_{h}-\left(\nabla \boldsymbol{v}_{h}\right) \boldsymbol{v}_{h}\right)-\nabla\left(p_{h}-q_{h}\right)\right), p_{e}^{K}\left(\lambda\left(\nabla \boldsymbol{w}_{h}\right) \boldsymbol{v}_{h}\right)\right)\right)_{K} \\
\leq & C \lambda \sum_{K \in \mathcal{T}_{h}} h_{K}^{2}\left\|\lambda\left(\left(\nabla \boldsymbol{u}_{h}\right) \boldsymbol{u}_{h}-\left(\nabla \boldsymbol{v}_{h}\right) \boldsymbol{v}_{h}\right)+\nabla\left(p_{h}-q_{h}\right)\right\|_{0, K}\left\|\boldsymbol{v}_{h}\right\|_{\infty, K}\left|\boldsymbol{w}_{h}\right|_{1, K} \\
\leq & C \lambda\left\{\lambda\left(\left\|\left(\boldsymbol{u}_{h}, p_{h}\right)\right\|+\left\|\left(\boldsymbol{v}_{h}, q_{h}\right)\right\|\right)+1\right\}\left\|\left(\boldsymbol{v}_{h}, q_{h}\right)\right\| \\
& \left\|\left(\boldsymbol{u}_{h}, p_{h}\right)-\left(\boldsymbol{v}_{h}, q_{h}\right)\right\|\left\|\left(\boldsymbol{w}_{h}, t_{h}\right)\right\| \\
\leq & C \lambda\{2 \lambda+1\}\left\|\left(\boldsymbol{u}_{h}, p_{h}\right)-\left(\boldsymbol{v}_{h}, q_{h}\right)\right\|\left\|\left(\boldsymbol{w}_{h}, t_{h}\right)\right\|
\end{aligned}
$$

and

$$
\begin{aligned}
\mathrm{IV} & =-\sum_{K \in \mathcal{T}_{h}}\left(p_{e}^{K}\left(\lambda\left(\nabla \boldsymbol{v}_{h}\right) \boldsymbol{v}_{h}-\lambda\left(\nabla \boldsymbol{u}_{h}\right) \boldsymbol{u}_{h}\right), p_{e}^{K}\left(\nabla t_{h}\right)\right)_{K} \\
& \leq C \lambda\left\|\left(\boldsymbol{u}_{h}, p_{h}\right)-\left(\boldsymbol{v}_{h}, q_{h}\right)\right\|\left\|\left(\boldsymbol{w}_{h}, t_{h}\right)\right\| .
\end{aligned}
$$

Collecting the bounds (B.2), (B.3), (B.4), and (B.5), inequality (B.1) becomes

$$
\begin{aligned}
\| T_{h} G_{h}\left(\lambda, \boldsymbol{u}_{h}, p_{h}\right)-T_{h} G_{h} & \left(\lambda, \boldsymbol{v}_{h}, q_{h}\right) \| \\
\leq & C \lambda\left\{4+3 \lambda+h\|\boldsymbol{f}\|_{0, \Omega}\right\}(1+\lambda h)^{2}\left\|\left(\boldsymbol{u}_{h}, p_{h}\right)-\left(\boldsymbol{v}_{h}, q_{h}\right)\right\|
\end{aligned}
$$

and thus the result follows under the assumption that $\lambda$ is such that $C \lambda\{4+3 \lambda+$ $\left.h\|\boldsymbol{f}\|_{0, \Omega}\right\}(1+\lambda h)^{2}<1$.

Acknowledgments. The authors wish to thank the anonymous referees whose questions and constructive criticism helped improve the quality of the present manuscript.

\section{REFERENCES}

[1] G. Barrenechea and F. Valentin, Consistent local projection stabilized finite element methods, SIAM J. Numer. Anal., 48 (2010), pp. 1801-1825.

[2] G. Barrenechea and F. Valentin, A residual local projection method for the Oseen equation, Comput. Methods Appl. Mech. Engrg., 199 (2010), pp. 1906-1921.

Copyright $@$ by SIAM. Unauthorized reproduction of this article is prohibited. 
[3] G. Barrenechea and F. Valentin, Beyond pressure stabilization: A low order local projection method for the Oseen equation, Internat. J. Numer. Methods Engrg., 86 (2011), pp. 801815.

[4] R. Becker And M. BraAck, A finite element pressure gradient stabilization for the Stokes equations based on local projections, Calcolo, 38 (2001), pp. 173-199.

[5] P. Bochev, C. R. Dohrmann, And M. D. Gunzburger, Stabilization of low-order mixed finite elements for the Stokes equations, SIAM J. Numer. Anal., 44 (2006), pp. 82-101.

[6] M. BRAACK AND E. BURMAN, Local projection stabilization for the oseen problem and its interpretation as a variational multiscale method, SIAM J. Numer. Anal., 6 (2006), pp. 25442566.

[7] M. BRAACK AND G. LuBe, Finite elements with local projection stabilization for incompressible flow problems, J. Comput. Appl. Math., 27 (2009), pp. 116-144.

[8] F. Brezzi, J. RAPPAZ, AND P. RAVIART, Finite dimensional approximation of non-linear problems I. Branches of non-singular solutions, Numer. Math., 36 (1980), pp. 1-25.

[9] P. Clément, Approximation by finite element functions using local regularization, RAIRO Anal. Numer., 9 (1975), pp. 77-84.

[10] R. Codina AND J. Blasco, Analysis of a pressure-stabilized finite element approximation of the stationary Navier-Stokes equations, Numer. Math., 87 (2000), pp. 59-81.

[11] C. Dohrman And P. Bochev, A stabilized finite element method for the Stokes problem based on polynomial pressure projections, Internat. J. Numer. Methods Fluids, 46 (2004), pp. 183-201.

[12] A. Ern and J.-L. Guermond, Theory and Practice of Finite Elements, Appl. Math. Sci. 159, Springer-Verlag, New York, 2004.

[13] L. Franca And S. Frey, Stabilized finite element methods: II. The incompressible NavierStokes equations, Comput. Methods Appl. Mech. Engrg., 99 (1992), pp. 209-233.

[14] L. Franca, C. Harder, And F. Valentin, On a residual local projection method for the Darcy equation, C. R. Acad. Sci. Paris, 347 (2009), pp. 1105-1110.

[15] S. Ganesan, G. Matthies, and L. Tobiska, Local projection stabilization of equal order interpolation applied to the Stokes problem, Math. Comp., 77 (2008), pp. 2039-2060.

[16] U. Ghia, K. Ghia, And C. Shin, High-Re solutions for incompressible flow using the NavierStokes equations and a multigrid method, J. Comput. Phys., 48 (1982), pp. 387-411.

[17] V. Girault and P. Raviart, Finite element methods for the Navier-Stokes equations: Theory and algorithms, Springer Ser. in Comput. Math. 5, Springer-Verlag, Berlin, 1986.

[18] G. Lube AND P. KNOBLOCH, Local projection stabilization for advection-diffusion-reaction problems: One-level vs. two-level approach, Appl. Numer. Math., 52 (2009), pp. 2891-2907.

[19] G. Medic and B. Mohammadi, NSIKE-An Incompressible Navier-Stokes Solver for Unstructured Meshes, Technical report, INRIA, 1999.

[20] H. Roos, M. Stynes, And L. Tobiska, Robust Numerical Methods for Singularly Perturbed Differential Equations, 2nd ed., Comput. Math. 24, Springer-Verlag, Berlin, 2008.

[21] M. Schäfer AND S. Turek, Benchmark computations of laminar flow around a cylinder, in Flow Simulation with High-Performance Computer II, E. Hirschel, ed., Notes Fluid Mechanics 52, Springer Vieweg, Weisbaden, 1996, pp. 547-566.

[22] R. Temam, Navier-Stokes Equations and Nonlinear Functional Analysis, SIAM, Philadelphia, 1995.

[23] V. Thomée, Galerkin Finite Element Methods for Parabolic Problems, 2nd ed., Springer Ser. Comput. Math. 25, Springer-Verlag, Berlin, 2006.

[24] L. TOBISKA AND R. VERFürTh, Analysis of a streamline diffusion finite element method for the Stokes and Navier-Stokes equations, SIAM J. Numer. Anal., 33 (1996), pp. 107-127.

[25] S. TureK, Efficient Solvers for Incompressible Flow Problems, Lecture Notes in Comput. Sci. Engrg. 6, Springer-Verlag, Berlin, 1999.

Copyright $@$ by SIAM. Unauthorized reproduction of this article is prohibited. 